\title{
Fair greening of broadband access: spectrum management for energy-efficient DSL networks
}

\author{
Paschalis Tsiaflakis $^{1 *}$, Yung Yi ${ }^{2}$, Mung Chiang ${ }^{3}$ and Marc Moonen ${ }^{1}$
}

\begin{abstract}
Dynamic spectrum management (DSM) is recognized as a promising technology to reduce power consumption in DSL access networks. However, the correct formulation of power-aware DSM problem statements requires a proper understanding of greening, i.e., reducing power consumption. In this paper, we, therefore, investigate greening and show that it can be decomposed into two dimensions: the price of greening and the fairness of greening. We first analyze the price of greening, providing theoretical bounds on the power-rate trade-off and identifying the typical trends that can be expected in practice, with some particularly promising results. Then, we introduce the fairness dimension, where we show that fairness becomes crucial when reducing power consumption. We propose four different fair greening policies that can be used to obtain a favorable trade-off between fast, fair and green DSL operation. Finally, we evaluate and quantify the corresponding trade-offs for realistic DSL access networks.
\end{abstract}

\section{Introduction}

Digital subscriber line (DSL) refers to a family of technologies that enable digital broadband Internet access over the local telephone network. It is currently the dominating wireline broadband access technology with a global market share of $65 \%$ [1]. The main reason for its popularity is its low deployment cost, as DSL reuses the twisted pairs of the existing telephone network infrastructure to connect subscribers to the Internet backbone.

To cope with the increasing demands of the users (endusers as well as service providers) and to stay competitive with other broadband access technologies, DSL technology is continuously innovated to further improve its broadband performance and so as to extend its life span. One of the major impairments that limits further improvement of DSL performance is crosstalk, i.e., the electromagnetic interference among different lines in the same cable bundle. The presence of crosstalk turns the DSL access network into a very challenging interference environment where the transmission of one line can significantly degrade the performance of the other lines. Proper network management of these interfering lines is, therefore, crucial to prevent huge performance degradations.

\footnotetext{
* Correspondence: paschalis.tsiaflakis@esat.kuleuven.be 'Department of Electrical Engineering, ESAT/SCD, Katholieke Universiteit Leuven, Kasteelpark Arenberg 10, 3001 Leuven-Heverlee, Belgium Full list of author information is available at the end of the article
}

Dynamic spectrum management (DSM) is recognized as a key technology for tackling this crosstalk problem $[2,3]$ by jointly coordinating the transmission of the users, i.e., lines, in the network. There exist two types of multiuser coordination: spectrum level coordination [4-12] and signal level coordination [13-16]. In this paper, the focus will be on spectrum level coordination, also referred to as spectrum management, spectrum balancing or multicarrier power control. More specifically, spectrum level coordination comes down to allocating the transmit spectrum, i.e., transmit powers over all frequencies, to the different users so as to prevent the destructive impact of crosstalk. It is already shown in literature that this level of access network coordination can spectacularly boost data rates $[2,3,5]$. Note that in this paper, DSM will refer to spectrum coordination.

Significant research efforts have been spent on DSM to increase the data rates in DSL access networks, i.e., rateadaptive DSM. However, only little attention has been devoted to true power minimization. Recently, power consumption has gained importance (e.g., ITU-T Study Group 15). Information and communication technologies (ICTs) have been identified as significant contributors to global warming [17]. Broadband equipment contributes to the electricity consumption, where a total European consumption of up to 50 TWh per year can be estimated for the year 2015 [18]. Therefore, the European Code of Conduct for Broadband Equipment takes initiative in 
setting up general principles and actions, and targets to limit the (maximum) electricity consumption to $25 \mathrm{TWh}$ per year, which is equivalent to 5.5 million ton of oil equivalent and to a total saving of about $€ 7.5$ billion per year. DSL, as the most widely deployed wireline broadband access technology today, plays an important role in this initiative [19]. The Broadband Forum encourages international standard bodies to develop techniques for power reduction within the scope of their activities and to maximize power savings while preserving and enhancing quality of service [19].

One of the technologies that fits well in this framework is DSM. More specifically, a large portion of the power consumption of today's state-of-the-art VDSL2 chipsets is due to the line driver [20]. The line driver consumption depends on the transmit powers. Now, DSM can be used to reduce the transmit powers, which is reflected into an overall power reduction.

In this paper, we, therefore, revisit DSM with a specific focus on how to approach greening, i.e., reducing power consumption, in DSL systems. Although some powerminimizing approaches have been proposed in literature recently, there is no thorough investigation into the concept of greening. What is greening and how should we analyze it properly? What are good greening strategies for (possibly very asymmetric) interference-limited systems? It is important to investigate the trade-off between both contradictory objectives, i.e., data rate maximization (fast) versus power minimization (green). Reducing power consumption results in a loss of data rate performance, which can be seen as the price of greening. The relation between greening and the price of greening depends on the crosstalk among the users and should be investigated for different practical settings so as to assess the potential of greening DSL broadband Internet access. Furthermore, we identify that there is a second crucial dimension in greening, namely fairness. We observe that straightforwardly reducing total system power may result in very unfair allocations. Therefore, the focus is also on providing concrete greening policies that strive toward obtaining fairness among the users, resulting in a desirable trade-off between fast, fair and green DSL operation. Note that the fairness part has a connection with the operator's service plans.

Power-efficient DSM is only a very recent topic in the field of DSL broadband access. However, the main focus up to now has been on algorithm design, where a number of parallel developments have been presented. In [4] iterative distributed DSM algorithms are proposed for minimizing the sum power for each user. In $[7,20-24]$ the problem of minimizing the (weighted) sum transmit powers of the multiuser DSL system has been considered, where different solution procedures are proposed: in $[20,24]$ procedures are proposed based on iterative geometric programming (GP) approximation; in [21,23], power back-off-based procedures are proposed; in $[22,25,26]$, solutions are proposed that make use of existing rate-adaptive DSM algorithms; in [27], power consumption is reduced and stability is improved by using a band-preference method. All the above research contributions on improving the power efficiency of DSL systems by the usage of DSM are understood to fall under a common term referred to as 'Green DSL'. We particularly also refer to $[25,26]$ in which a general Green DSL framework was proposed. Besides the field of DSL systems, power-efficient resource allocation has also been considered in different contexts such as for beamforming [28] and also for fading multiple-access and broadcast channels [29] in wireless contexts.

Existing recent work has thus mainly focused on algorithm design for power-minimizing DSM. This paper, however, focuses on the greening system modeling part, how to analyze greening and the impact and potential of greening in DSL broadband access. More specifically, the main contributions of this paper are as follows:

(i) First, we show how existing work on power-minimizing DSM can be framed into a general Green DSL framework that covers all existing DSM problem formulations. We then propose a number of novel power-aware DSM problem formulations, next to a first energy-aware DSM problem formulation that focuses on energies instead of on transmit powers. This general Green DSL framework is then explained using the duality between rate regions and power regions, which results in a simple but powerful geometric interpretation of power-aware DSM.

(ii) Within this geometric setting, we identify that greening can be understood and decomposed in two different dimensions: (1) the price of greening and (2) the fairness of greening. Both dimensions reflect different design objectives in a multiuser setting and should be properly taken into account when designing greening strategies.

(iii) The 'price of greening dimension' is then investigated for DSL scenarios. Next to a theoretical worst-case analysis, we quantify the power-rate trade-off for different practical settings. We also investigate the properties that determine these promising power-rate trade-offs and identify the trends that can be expected for practical DSL systems.

(iv) We then investigate the second dimension of greening, which is the 'fairness dimension', and identify that fairness becomes a crucial design objective when applying greening. We introduce the concept of fair greening, which allows to consider fairness when allocating the price of greening over the DSL users. Four concrete greening policies are proposed to 
obtain fair greening in future DSL networks, and their practical impact and potential for realistic DSL scenarios is evaluated. Note that fairness has been studied thoroughly in the context of data rates [30,31]. However, fairness in the context of reducing energy is a novel concept that has, as far as we know, not been studied before.

The rest of the paper is organized as follows. In Section 2 , the system model is introduced with some background information on 'DSM design'. In Section 3, the 'Green DSL' framework from [26] is briefly summarized, and novel power and energy-aware DSM formulations are proposed. This is then framed within a rate and power region setting. In Section 4, the concept of greening is analyzed in which the two dimensions 'the price of greening' and 'the fairness of greening' are introduced. These dimensions are further investigated and evaluated with realistic DSL systems in Sections 4-B and 4-C, respectively. We would like to highlight here that although the concepts and ideas will be explained for DSL wireline communications, they can also be applied to OFDM-based wireless greening.

\section{System model and background: DSM design}

\section{A. System model}

We consider a network, i.e., cable bundle, consisting of $\mathcal{N}=\{1, \ldots, N\}$ interfering DSL users (i.e., lines, modems) with the standard assumption of synchronous discrete multitone (DMT) modulation with $\mathcal{K}=\{1, \ldots, K\}$ tones (i.e., frequency carriers). The transmission can then be modeled independently on each tone $k$ as follows:

$$
\mathbf{y}_{k}=\mathbf{H}_{k} \mathbf{x}_{k}+\mathbf{z}_{k}, \quad k \in \mathcal{K} .
$$

The vector $\mathbf{x}_{k}=\left[x_{k}^{1}, \ldots, x_{k}^{N}\right]^{\mathrm{T}}$ contains the transmitted signals on tone $k$, where $x_{k}^{n}$ refers to the signal transmitted by user $n$ on tone $k$. Vectors $\mathbf{z}_{k}$ and $\mathbf{y}_{k}$ have similar structures; $\mathbf{z}_{k}$ refers to the additive noise on tone $k$, containing thermal noise, alien crosstalk and radio frequency interference (RFI); $\mathbf{y}_{k}$ refers to the received signals on tone $k . \mathbf{H}_{k}$ is an $N \times N$ matrix with $\left[\mathbf{H}_{k}\right]_{n, m}=h_{k}^{n, m}$ referring to the channel gains from transmitter $m$ to receiver $n$ on tone $k$. The diagonal elements are the direct channels, and the off-diagonal elements are the crosstalk channels.

The transmit power of user $n$ on tone $k$, also referred to as transmit power spectral density, is denoted as $s_{k}^{n} \triangleq \Delta_{f} E\left\{\left|x_{k}^{n}\right|^{2}\right\}$, where $\Delta_{f}$ refers to the tone spacing. The vector $\mathbf{s}_{k} \triangleq\left\{s_{k}^{n}, n \in \mathcal{N}\right\}$ denotes the transmit powers of all users on tone $k$. The vector $\mathbf{s}^{n} \triangleq\left\{s_{k}^{n}, k \in \mathcal{K}\right\}$ denotes the transmit powers of user $n$ on all tones, i.e., the transmit spectrum of user $n$. The vector $\mathbf{s} \triangleq\left\{s_{k}^{n}, k \in \mathcal{K}, n \in \mathcal{N}\right\}$ denotes all transmit powers in the network, i.e., of all users and over all tones. The received noise power by user $n$ on tone $k$, also referred to as noise spectral density, is denoted as $\sigma_{k}^{n} \triangleq \Delta_{f} E\left\{\left|z_{k}^{n}\right|^{2}\right\}$.

Note that we assume no signal coordination at the transmitters and at the receivers. The system model thus corresponds to a multitone interference channel where the interference is treated as additive white Gaussian noise. Under this standard assumption, the bit loading, i.e., bit rate, for user $n$ on tone $k$, given the transmit spectra $\mathbf{s}_{k}$ of all users on tone $k$, is

$$
b_{k}^{n}=b_{k}^{n}\left(\mathbf{s}_{k}\right) \triangleq \log _{2}\left(1+\frac{1}{\Gamma} \frac{\left|h_{k}^{n, n}\right|^{2} s_{k}^{n}}{\sum_{m \neq n}\left|h_{k}^{n, m}\right|^{2} s_{k}^{m}+\sigma_{k}^{n}}\right) \text { bits/Hz, }
$$

in which $\Gamma$ denotes the SNR gap to capacity, which is a function of the desired BER, the coding gain and noise margin [32]. The DMT symbol rate is denoted as $f_{s}$. The achievable total data rate $R^{n}$ for user $n$ and the total transmit power $P^{n}$ consumed by user $n$ can now be expressed in function of the transmit powers $s_{k}^{n}, n \in \mathcal{N}, k \in \mathcal{K}$, as follows:

$$
R^{n} \triangleq f_{s} \sum_{k \in \mathcal{K}} b_{k}^{n}\left(\mathbf{s}_{k}\right) \quad \text { and } \quad P^{n} \triangleq \sum_{k \in \mathcal{K}} s_{k}^{n} .
$$

We would like to highlight that $R^{n}$ corresponds to a challenging nonconvex function of the transmit powers $s_{k}^{n}, n \in \mathcal{N}, k \in \mathcal{K}$.

\section{B. DSM design}

The basic concept of DSM through network level spectrum coordination is to allocate the transmit powers over all users and tones, i.e. $s_{k}^{n}, n \in \mathcal{N}, k \in \mathcal{K}$, by taking the specific physical channel conditions (channel gains and noise) into account, and so as to pursue certain design objectives and/or satisfy certain constraints. A number of transmit power constraints are standardly imposed by DSL standards. More specifically, there is an upper bound on the total transmit power budget that each user $n$ can allocate over its tones as follows

$$
P^{n} \leq P^{n \text {,tot }}, n \in \mathcal{N},
$$

in which $P^{n \text {,tot }}$ refers to the total available power budget for user $n$. There are also constraints on the transmit power allocated into each tone, which are referred to as spectral mask constraints, and are characterized by the following set:

$$
\mathcal{S}=\left\{\left(s_{k}^{n}: n \in \mathcal{N}, k \in \mathcal{K}\right): 0 \leq s_{k}^{n} \leq s_{k}^{n, \text { mask }}, n \in \mathcal{N}, k \in \mathcal{K}\right\},
$$

in which $s_{k}^{n \text {,mask }}$ refers to the spectral mask constraint for user $n$ on tone $k$.

The set of all possible data rate allocations, which satisfy the constraints (3) and (4), can be characterized 
by the achievable rate region $\mathcal{R}$ :

$$
\begin{array}{r}
\mathcal{R}=\left\{\left(R^{n}: n \in \mathcal{N}\right) \mid R^{n}=f_{s} \sum_{k \in \mathcal{K}} b_{k}^{n}\left(\mathbf{s}_{k}\right), n \in \mathcal{N},\right. \\
\text { with } \left.P^{n} \leq P^{n, \text { tot }}, n \in \mathcal{N}, \text { and } \mathbf{s} \in \mathcal{S}\right\} .
\end{array}
$$

Note that this rate region can be assumed to be convex for the considered multitone interference channel as the number of tones $K$ is typically very large $[5,33]$.

A typical design objective $[4-9,12]$, is to optimize the transmit powers so as to achieve some Pareto-optimal allocation of data rates $R^{n}, n \in \mathcal{N}$, somewhere on the boundary of the achievable rate region. However, other design objectives are also possible, considering other performance measures like transmit power, fairness, delay, etc. In general, we will refer to a DSM design as an optimization problem where the transmit powers correspond to the optimization variables, and where the objectives and/or constraints are functions of these transmit powers and reflect the quality of service (QoS) requirements for the corresponding DSL network. Note that the transmit power constraints (3) and (4) are required in all DSM designs in order to be compliant with DSL standards.

\section{GREEN DSL}

\section{A. Green DSL framework}

Research efforts in DSM have mainly focused on DSM designs involving purely data rate driven objectives [4-9,12], to achieve some Pareto-optimal allocation of data rates subject to power constraints (3) and (4). These DSM designs, however, typically correspond to a very inefficient power usage. For instance, one of the first proposed DSM designs is (6) in which the data rate of one user $R^{1}$ is maximized subject to minimum data rate constraints for the other users, i.e., $R^{n} \geq R^{n, \text { target }}, n>1$, and user total transmit power constraints, i.e., $P^{n} \leq P^{n \text {, tot }}$, $n \in \mathcal{N}$, with $\mathbf{R}^{\text {target }}=\left[R^{1, \text { target }}, \ldots, R^{\mathrm{N}, \text { target }}\right]^{\mathrm{T}}$, and $\mathbf{P}^{\text {tot }}=$ $\left[P^{1, \text { tot }}, \ldots, P^{\mathrm{N}, \text { tot }}\right]^{\mathrm{T}}$.

$$
\begin{aligned}
\underset{\mathbf{s} \in \mathcal{S}}{\operatorname{maximize}} R^{1} & \\
\text { subject to } & R^{n} \geq R^{n, \text { target }}, \quad n \in \mathcal{N}>1, \\
& P^{n} \leq P^{n \text {,tot }, \quad n \in \mathcal{N} .}
\end{aligned}
$$

Another popular DSM design is the maximization of a weighted sum of data rates, as follows

$$
\begin{aligned}
& \underset{s \in \mathcal{S}}{\operatorname{maximize}} \sum_{n \in \mathcal{N}} w_{n} R^{n} \\
& \text { subject to } P^{n} \leq P^{n, \text { tot }}, \quad n \in \mathcal{N},
\end{aligned}
$$

in which the weights $\mathbf{w}=\left[w_{1}, \ldots, w_{N}\right]$ are used to give more priority to some users with respect to the other users. This formulation also plays a central role in many other network control and optimization methods, such as, for instance, crosslayer control policies in wireless networks $[34,35]$.

Recently, also a limited number of energy-aware DSM designs have been proposed $[7,20,22,24]$, such as the minimization of the (weighted) sum of transmit powers subject to data rate constraints

$$
\begin{aligned}
\underset{\mathbf{s} \in \mathcal{S}}{\operatorname{minimize}} & \sum_{n \in \mathcal{N}} t_{n} P^{n} \\
\text { subject to } & R^{n} \geq R^{n \text {,target }}, \quad n \in \mathcal{N}, \\
& P^{n} \leq P^{n, \text { tot },} \quad n \in \mathcal{N},
\end{aligned}
$$

in which $\mathbf{t}=\left[t_{1}, \ldots, t_{N}\right]$ are weights.

A general Green DSL framework can now be formed that models DSM designs, as we showed in our recent work [26]. It allows to model different trade-offs between maximizing data rates (fast) and reducing the system power consumption (green). It corresponds to a general optimization problem in which the objectives and constrains are expressed as a function of the individual data rates $\mathbf{R}=\left[R^{1}, \ldots, R^{N}\right]^{\mathrm{T}}$ and total powers $\mathbf{P}=$ $\left[P^{1}, \ldots, P^{N}\right]^{\mathrm{T}}$, and in which the optimization variables are the transmit powers over all users and tones $s_{k^{\prime}}^{n} n \in \mathcal{N}, k \in \mathcal{K}_{\text {, }}$ under spectral mask constraints $\mathcal{S}$, as follows:

$$
\begin{array}{r}
\underset{\mathbf{s} \in \mathcal{S}}{\operatorname{maximize}} U(\mathbf{R}, \mathbf{P}) \\
\text { subject to } I(\mathbf{R}, \mathbf{P}) \leq 0, \\
E(\mathbf{R}, \mathbf{P})=0,
\end{array}
$$

with the objective function $U(\mathbf{R}, \mathbf{P}): \mathbb{R}_{+}^{N} \times \mathbb{R}_{+}^{N} \rightarrow \mathbb{R}$, the inequality constraints $\mathbf{I}(\mathbf{R}, \mathbf{P}): \mathbb{R}_{+}^{N} \times \mathbb{R}_{+}^{N} \rightarrow \mathbb{R}^{M}$, the equality constraints $\mathbf{E}(\mathbf{R}, \mathbf{P}): \mathbb{R}_{+}^{N} \times \mathbb{R}_{+}^{N} \rightarrow \mathbb{R}^{L}$, and in which $M$ denotes the number of inequality constraints and $L$ the number of equality constraints. Although typical DSM designs are generally NP-hard nonconvex problems, efficient dual-decomposition-based procedures have been proposed in [25,26,36] for tackling Green DSL DSM designs.

\section{B. Novel power-aware DSM designs}

Using the general Green DSL framework of [26], we can now define a whole range of novel DSM designs that model different trade-offs between data rates and transmit power consumption.

For instance, one may put a constraint on the sum of all allocated transmit powers so as to reduce the total consumed transmit power by a factor $\alpha$, i.e.,

$$
\begin{aligned}
\underset{\mathbf{s} \in \mathcal{S}}{\operatorname{maximize}} & \sum_{n \in \mathcal{N}} w_{n} R^{n} \\
\text { subject to } & R^{n} \geq R^{n, \text { target }}, \quad n \in \mathcal{N}, \\
& P^{n} \leq P^{n, \text { tot },} \quad n \in \mathcal{N}, \\
& \sum_{n \in \mathcal{N}} P^{n} \leq \alpha \sum_{n \in \mathcal{N}} P^{n, \text { tot }},
\end{aligned}
$$


where $0<\alpha \leq 1$ is a chosen constant and denotes the desired power reduction with respect to full transmit power usage. This formulation can be relevant when a service provider aims to reduce the network transmit power consumption by a factor $\alpha$.

One can also pursue a specific proportion in terms of the total transmit power consumed by the individual users, leading to following DSM design:

$$
\begin{aligned}
\underset{P, \mathbf{s} \in \mathcal{S}}{\operatorname{minimize}} P & \\
\text { subject to } & P^{n} \leq \gamma_{n} P, \quad n \in \mathcal{N}, \\
& R^{n} \geq R^{n, \text { target }}, \quad n \in \mathcal{N}, \\
& P^{n} \leq P^{n, \text { tot }}, \quad n \in \mathcal{N},
\end{aligned}
$$

where the $\left\{\gamma_{n}: n \in \mathcal{N}\right\}$ indicate the proportions with respect to a base power $P$. One interesting objective would, for instance, be to minimize the maximum of all transmit powers $P^{n}$, which corresponds to (11) with $\gamma_{n}=1, n \in \mathcal{N}$.

Power-efficient network operation may also be achieved by maximizing the minimum user ratio of the data rate to its total transmit power, i.e., [bits/Watts], as follows:

$$
\begin{aligned}
\underset{\beta, \mathbf{s} \in \mathcal{S}}{\operatorname{maximize}} \beta & \\
\text { subject to } & R^{n} \geq R^{n, \text { target }}, \quad n \in \mathcal{N}, \\
& P^{n} \leq P^{n, \text { tot }}, \quad n \in \mathcal{N}, \\
& \frac{R^{n}}{P^{n}} \geq \beta, \quad n \in \mathcal{N},
\end{aligned}
$$

with a nonlinear third constraint.

\section{Energy-aware DSM design}

Instead of the typically transmit power-based DSM formulations for minimizing transmit power consumption, we will now propose a first energy-driven DSM formulation. For this, we start from a different setting in which a known set of jobs $J^{n}$, i.e., data, is to be transmitted by each user $n$, and we try to minimize the consumed energy for finishing these jobs. The consumed energy for finishing the job $J^{n}$ by user $n$ can be expressed as $E^{n}=P^{n} T^{n}$, with $T^{n}$ being the time required to finish the job $J^{n}$, i.e., $T^{n}=J^{n} /$ $R^{n}$. This results in the following energy-driven DSM formulation

$$
\begin{aligned}
\underset{\mathbf{s} \in \mathcal{S}}{\operatorname{minimize}} & \sum_{n \in \mathcal{N}} E^{n} \\
\text { subject to } & T^{n} \leq T^{n, \text { target }}, \quad n \in \mathcal{N}, \\
& P^{n} \leq P^{n \text {,tot },} \quad n \in \mathcal{N},
\end{aligned}
$$

in which the first set of constraints corresponds to time deadlines $T^{n \text {,target }}$, before which the jobs need to be finished. In "Appendix A", we prove that this problem statement can be reformulated as (8) with weights $t_{n}=$ $T^{n \text {, target }}$ and constraints $R^{n}=R^{n \text {,target }}=J^{n} / T^{n \text {, target }}$ and can thus be readily solved using the Green DSL algorithms described in $[25,26,36]$. The main message for energy minimization is to transmit as slow as possible tightly satisfying the timing constraints.

\section{A geometric view of green DSL by achievable rate and power regions}

All the previous DSM formulations can now be characterized visually using the achievable rate region $\mathcal{R}$ and the achievable power region $\mathcal{P}$, which are shown in Figure 1 for a two-user DSL scenario. The concept of the power region has already been introduced in other domains, e.g., for resource allocation in wireless networks [29]. The boundary of the (achievable) rate region is defined as the Pareto-optimal trade-off in achievable data rates for fixed maximum power budgets $\mathbf{P}^{\text {tot }}$ for the users. Similarly the boundary of the power region can be defined as the Pareto-optimal trade-off in user total transmit powers for fixed minimum data rate constraints $\mathbf{R}^{\text {target }}$.

The working points of the different DSM designs (6)(13) are indicated and explained in Figure 1. Note that the data rate driven DSM designs (6)(7) can only characterize a working point on the boundary of the rate region, not a working point inside the rate region. These boundary points mostly correspond to transmit power allocations where all users fully use their available transmit power. Obviously, this is not the best strategy in terms of energy efficiency. In contrast, the poweraware DSM designs (8)-(13) also allow to achieve working points strictly inside the rate region, corresponding to more power-efficient working points inside the power region.

\section{Analysis of greening concept in green DSL A. Greening dimensions}

Greening is not just choosing any power/energy-aware DSM formulation. To show that a proper choice of DSM formulation is crucial in obtaining good greening strategies, we will analyze the concept of greening from a geometric point of view departing from the achievable rate and power regions. For the sake of clarity (without loss of generality), we first focus on a two-user case. As shown in Figure 2, a proportional decrease in transmit powers $\Delta \mathbf{P}$ corresponds to a reduction in data rates $\Delta \mathbf{R}$.

We define two dimensions $\mathbf{g}$ and $\mathbf{f}$ as follows

$$
\begin{aligned}
& \mathbf{g}=-\mathbf{w} \eta \\
& \mathbf{f}=\mathbf{v} \zeta
\end{aligned}
$$

with $\eta$ and $\zeta$ scalar multiples, and vector $\mathbf{v}$ orthogonal to the rate vector before greening $\mathbf{R}^{o}$.

Dimension $\mathbf{g}$ lies along the direction of the weight $\mathbf{w}$ (of (7)) in $\mathbf{R}^{o}$ and can be seen as the negative gradient of the rate region, i.e., the direction in which the 

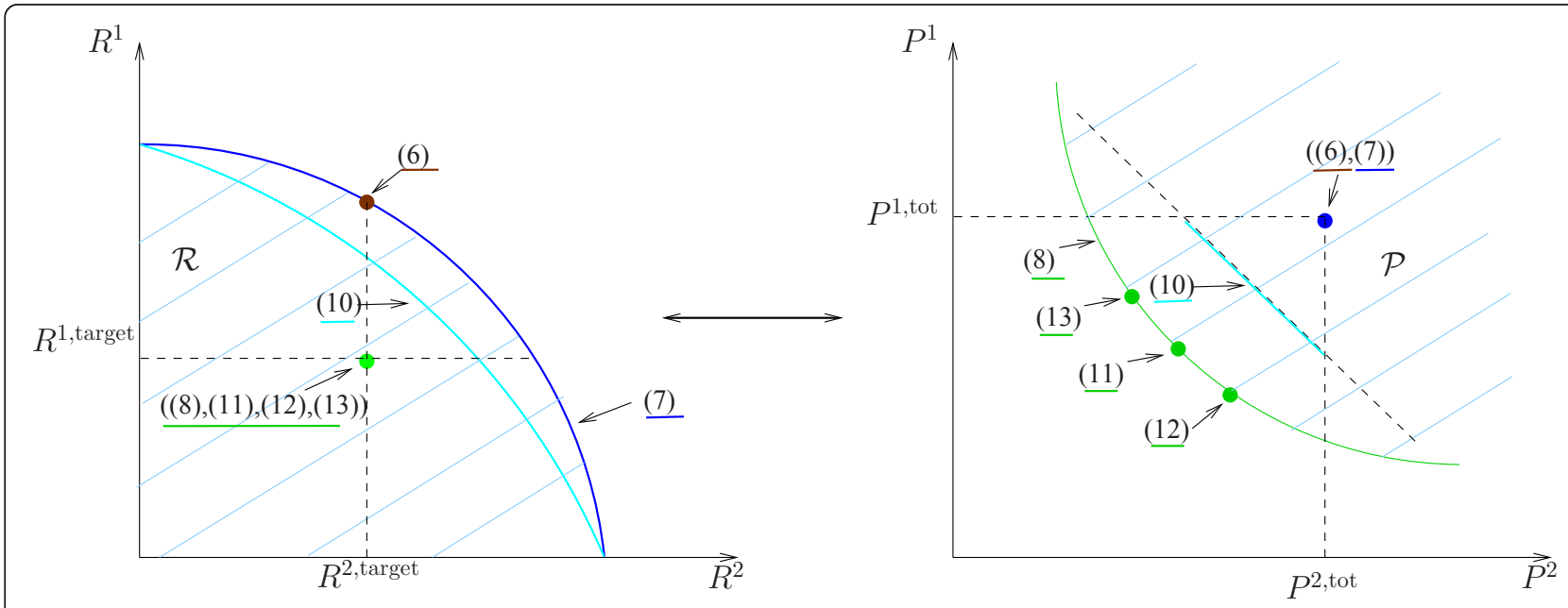

Figure 1 Characterization of working points in the achievable rate region (left) and achievable power region (right) for DSM designs (6)-(13). DSM designs (6) and (8) can be situated anywhere on the boundary of the rate region and power region, respectively, depending on the chosen weights $w_{n}$ and $t_{n}$ that reflect the relative importance of the users $n$. DSM design (10) corresponds to a reduced rate region as $\alpha<1$. DSM designs (8)(11)(12)(13) are all Pareto-optimal in power as they correspond to power minimizing DSM designs subject to minimum data rate constraints, i.e., DSM design (8) with a specific set of weights $t_{n}$.

weighted rate sum decreases the fastest. This dimension indicates the 'price of greening'.

Dimension $\mathbf{f}$ is orthogonal to the direction in which the data rates are reduced proportionally in $\mathbf{R}^{o}$. This dimension gives the direction in which data rates are distributed unfairly, and it thus indicates the 'fairness' dimension.

A particular data rate reduction $\Delta \mathbf{R}$ for given power reductions $\Delta \mathbf{P}$, i.e., greening vector, can now be decomposed into these two dimensions so as to understand how green and how fair a particular greening policy performs. More specifically, we can define the projection of a particular data rate reduction $\Delta \mathbf{R}$ on these two dimensions as follows

$$
\begin{aligned}
& \mathcal{P}_{\mathrm{g}}(\Delta \mathbf{R})=\text { the price of greening, } \\
& \mathcal{P}_{\mathrm{f}}(\Delta \mathbf{R})=\text { the fairness of greening, }
\end{aligned}
$$

which are also shown in Figure 2.

These two dimensions are essential in understanding greening. For instance, data rate reduction $\Delta R^{A}$ has a small component along $\mathbf{g}$ and thus corresponds to a small price of greening, i.e., a good power-rate trade-off. In other words, the distance to the boundary of the rate region before greening is small. However, the data rate
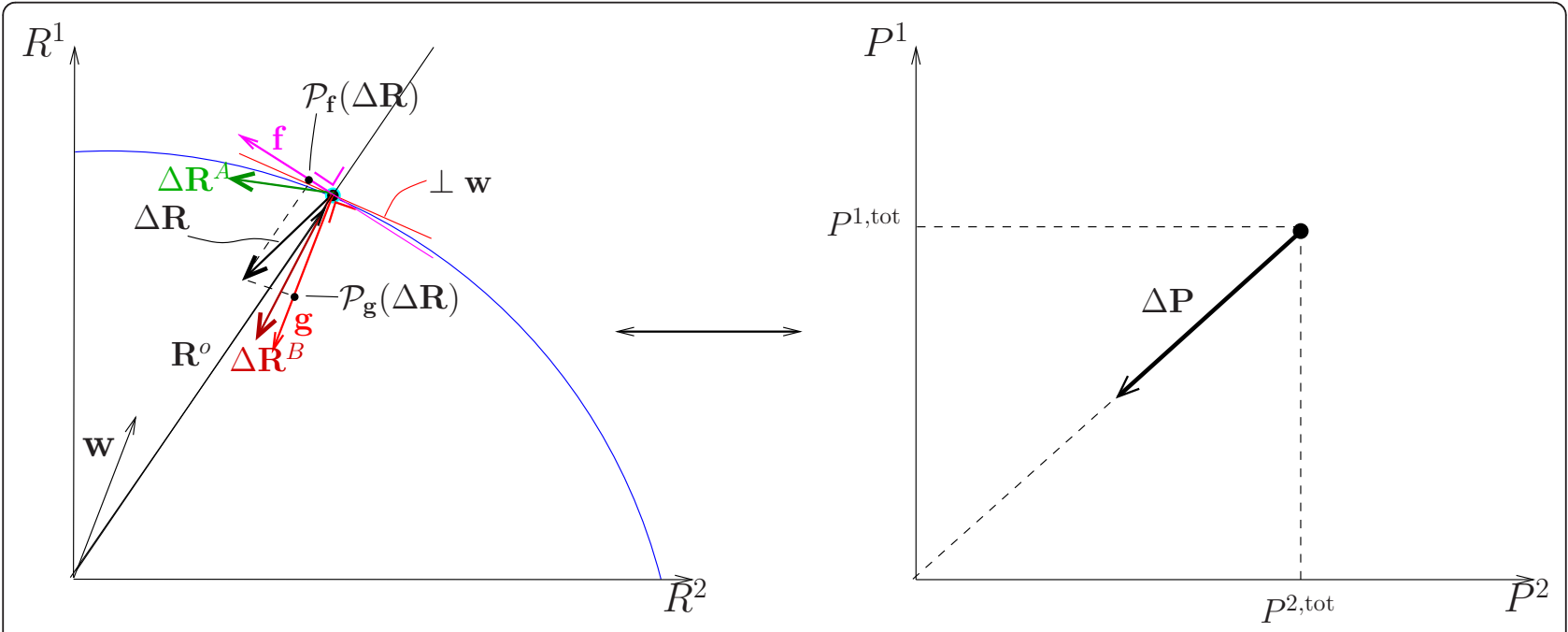

Figure 2 Greening vector $\Delta \mathrm{P}$ can result in different rate reductions $\Delta \mathrm{R}, \Delta \mathbf{R}^{A}$ and $\Delta \mathrm{R}^{B}$. $\Delta \mathrm{R}$ can be decomposed into two dimensions ('price of greening' dimension $g$ and fairness dimension f) given by the projections $\mathcal{P}_{\mathbf{g}}(\Delta \mathbf{R})$ and $\mathcal{P}_{\mathbf{f}}(\Delta \mathbf{R})$, respectively. 
distribution over the two users is very unfairly, i.e., user 2 reduces its data rate a lot, whereas user 1 even increases its data rate. In contrary, data rate reduction $\Delta R^{B}$ has a large price of greening, but a very fair (proportional) distribution of the data rates. In the ideal case, we should have small components along both dimensions.

In Figure 3, four rate reductions are shown for a given greening power reduction $\Delta \mathbf{P}$. In fact, the dashed rate region corresponds to all trade-offs in data rates that correspond to the reduced transmit powers, i.e., after greening. Depending on the greening method, we obtain different projections on the dashed rate region, corresponding to different rate reductions. $\Delta \mathbf{R}^{D}$ corresponds to the data rate reduction with the best weighted rate sum (from DSM design (7) with weight w). However, it has a very bad fairness performance. $\Delta \mathbf{R}^{B}$ is perfectly fair in data rate distribution, but it has a larger price of greening. $\Delta \mathbf{R}^{C}$ has a better fairness performance than $\Delta \mathbf{R}^{D}$ and a smaller price of greening than $\Delta \mathbf{R}^{B}$, demonstrating a trade-off between both dimensions. However, there is not always a trade-off as $\Delta \mathbf{R}^{A}$ performs worse than $\Delta \mathbf{R}^{B}$ in both dimensions.

A good greening formulation should thus focus on both dimensions, to obtain a good power-rate trade-off and also to prevent that weak users have to pay all data rate performance loss. Current DSM formulations do not consider these dimensions and consequently can result in very unfair or bad power-rate trade-offs, depending on the considered DSL scenario.

The previous two-user case can be straightforwardly extended to a general $\mathrm{N}$-user case, for which dimension $\mathbf{g}$ is unchanged (one-dimensional) and dimension $\mathbf{f}$

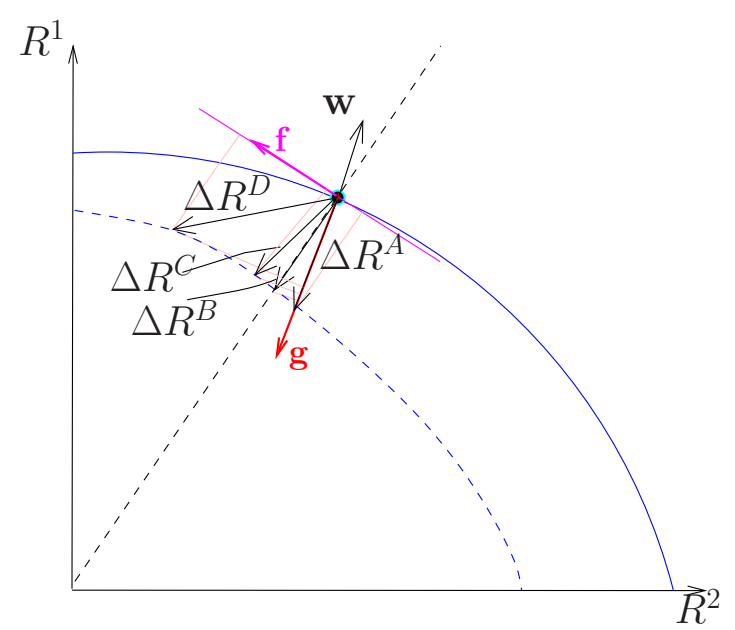

Figure 3 Different possible rate reductions for given greening $\Delta \mathbf{P}$ of Fig. 2. $g$ and $f$ are the 'price of greening' and 'fairness' dimensions, respectively. becomes an $N$-1-dimensional subspace orthogonal to vector $\mathbf{R}^{o}$.

In the following two sections, we will analyze DSL systems along the two dimensions $\mathbf{g}$ and $\mathbf{f}$. This will allow to identify good greening strategies and also to assess the potential of greening DSL broadband Internet access.

\section{B. Power-rate trade-off in green DSL}

The price of greening dimension, i.e., the power-rate trade-off, is an important greening dimension as it reflects the impact of saving power. The relation between transmit powers and data rates is, however, governed by very a nonconvex function, given by (1) and (2), which significantly complicates the theoretical analysis of the power-rate trade-off. Furthermore, the channel parameters depend heavily on the considered DSL setting. Therefore, a theoretical analysis that holds for all possible channel parameters does not allow to capture the particular structure of different practical settings.

In this section, we will, therefore, first provide theoretical lower and upper bounds on the optimal power-rate trade-off for a network without interference. It is then discussed how these bounds extend to the interference case. Based on these insights, we assess the price of greening for different practical settings by numerical simulations using practical channel models. This will allow to identify the typical trends that can be expected in DSL access systems under different practical settings.

\section{1) Theoretical analysis of power-rate trade-off}

We start with a multitone single-user bound analysis:

Lemma 4.1: For a single-user DSL system, the relative reduction in optimal data rate for a given relative reduction in transmit power of factor $c$, i.e., $f(c)$, is given by the expression:

$$
\begin{aligned}
f(c)= & \frac{R^{n}\left(c \cdot P^{n, \text { tot }}\right)}{R^{n}\left(P^{n, \text { tot }}\right)} \\
= & \frac{\sum_{k \in \mathcal{K}} \log _{2}\left(\frac{c}{K} P^{n, \text { tot }} \cdot \mathrm{CNR}_{k}^{n}+\frac{1}{K} \sum_{q \in \mathcal{K}} \frac{\mathrm{CNR}_{k}^{n}}{\mathrm{CNR}_{q}^{n}}\right)}{\sum_{k \in \mathcal{K}} \log _{2}\left(\frac{1}{K} P^{n, \text { tot }} \cdot \mathrm{CNR}_{k}^{n}+\frac{1}{K} \sum_{q \in \mathcal{K}} \frac{\mathrm{CNR}_{k}^{n}}{\mathrm{CNR}_{q}^{n}}\right)}
\end{aligned}
$$

with $R^{n}(P)$ being the optimal data rate for given total power $P$ and $\mathrm{CNR}_{k}^{n}=\left|h_{k}^{n, n}\right|^{2} /\left(\Gamma \sigma_{k}^{n}\right)$.

Proof: See "Appendix B".

Note that for the multiuser setting, we will use CNR to refer to the channel to interference and noise ratio, $\mathrm{i}$. e., $\mathrm{CNR}_{k}^{n}=\left|h_{k}^{n, n}\right|^{2} /\left(\Gamma\left(\sum_{m \neq n}\left|h_{k}^{n, m}\right|^{2} s_{k}^{m}+\sigma_{k}^{n}\right)\right)$.

Lemma 4.1 shows how the power-rate trade-off, i.e., $f$ (c) in function of $c$, depends on the CNRs, the transmit power budget and the power saving factor $c$. Using this 
lemma, we can obtain the following theorem describing the theoretical bounds on the power-rate trade-off.

Theorem 4.1: The power-rate trade-off, i.e., $f(c)$ in function of $c$, for a single-user DSL system is in the worst case linear. This linear relation is attained if all channel-to-noise ratios (CNRs) or the transmit power budget $P^{n \text {,tot }}$ go to zero. In contrary, when the CNRs or $P^{n, \text { tot }}$ go to infinity, the trade-off vanishes. Formally:

$$
\begin{aligned}
& \lim _{k \in \mathcal{K}: C N R_{k}^{n} \rightarrow 0} f(c)=c, \quad \text { and } \lim _{k \in \mathcal{K}: C N R_{k}^{n} \rightarrow \infty} f(c)=1, \\
& \lim _{P^{n, \text { ot } \rightarrow 0}} f(c)=c, \quad \text { and } \lim _{P^{n, \text { tot } \rightarrow \infty}} f(c)=1 .
\end{aligned}
$$

Proof: The proof follows trivially from Lemma 4.1 by taking a limit operation of (16).

Lemma 4.1 and Theorem 4.1 provide lower and upperbounds on the optimal power-rate trade-off that hold for any single-user DSL system setting. Furthermore, they show that the trade-off depends on the CNRs and the transmit power budget: larger CNRs and a larger transmit power budget result in a more favorable power-rate trade-off, as more power can be saved for a certain data rate decrease.

When extending these bounds to the multiuser interference case, one would at first sight expect that adding crosstalk to the noise reduces the overall CNRs and thus results in a less favorable power-rate trade-off, i.e., being more linear. However, crosstalk interference also results in a data rate decrease with respect to the single-user system, which has an impact on the relative data rate decrease for given power saving, i.e., $f(c)$. It turns out that the presence of crosstalk actually improves the power-rate trade-off. To demonstrate this, in Figure 4, we plot the trade-off between data rate performance and

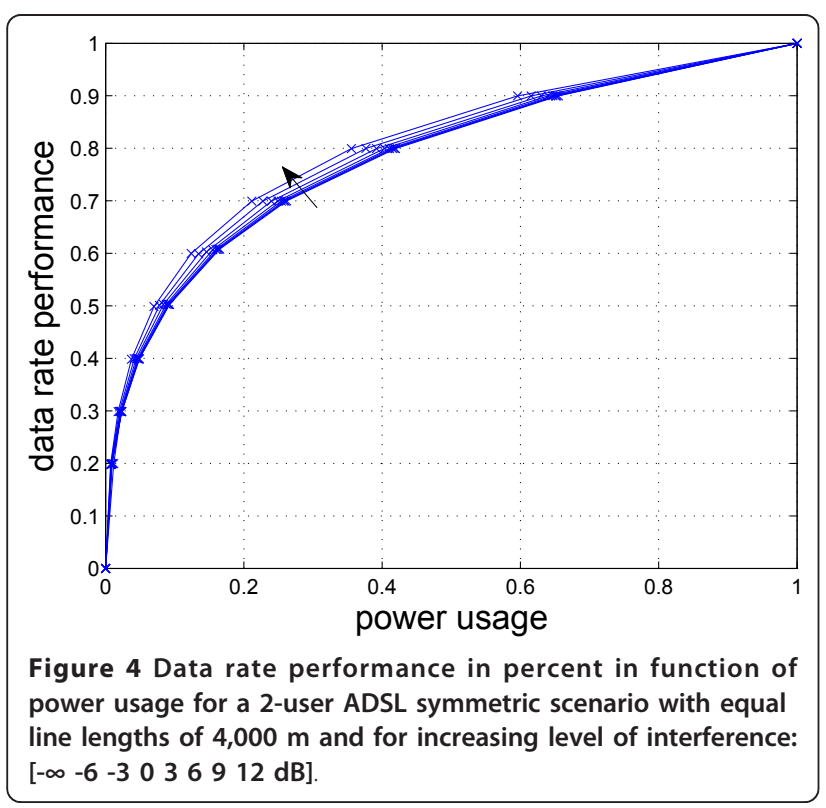

power usage for a 2-user ADSL symmetric scenario with equal line lengths of $4,000 \mathrm{~m}$, and where the crosstalk is simply scaled with factors ranging from 0 (i.e., no crosstalk) to $1 / 4,1 / 2,1,2,4,8$ and 16 . It can be observed that as crosstalk increases, the power-rate trade-off becomes more favorable, i.e., more power can be saved for a given data rate decrease.

We can give a very reasonable intuitive explanation as follows: in an access network with a lot of crosstalk, it requires a relatively larger amount of power to increase the data rate by a factor two, e.g., from 1 to 2 Mbps. This also means that in this network, reducing the data rate from 2 to $1 \mathrm{Mbps}$, will result in a larger relative power decrease than for a network with less crosstalk. Another explanation can be obtained from [33] in which it is shown that for the multiuser case and when the number of tones is large, the (un)weighted data rates show a socalled time sharing property in function of transmit powers. This means that the relation between the data rates and the total transmit powers is concave and thus in the worst case linear.

Given the above arguments, we can conclude that the upper and lower bounds of Theorem 4.1 also hold for the multiuser case, and we furthermore understand the factors that determine the power-rate trade-off.

\section{2) Simulation-based analysis of the power-rate trade-off}

The bounds of Section 4-B1 are valid for general channel parameters. They, however, do not capture the particular characteristics of typical practical settings. In this section, we will, therefore, assess these bounds for concrete DSL settings and investigate trends that can be expected for practical DSL systems using a simulationbased approach.

Simulation Setup The following realistic parameters settings are assumed for the DSL scenarios. The twisted pair lines have a diameter of $0.5 \mathrm{~mm}$ (24 AWG). The maximum transmit power is $20.4 \mathrm{dBm}$ for the ADSL scenarios and $11.5 \mathrm{dBm}$ for the VDSL scenarios. The SNR gap $\Gamma$ is $12.9 \mathrm{~dB}$, corresponding to a coding gain of $3 \mathrm{~dB}$, a noise margin of $6 \mathrm{~dB}$ and a target symbol error probability of $10^{-7}$. The tone spacing $\Delta_{f}$ is $4.3125 \mathrm{kHz}$. The DMT symbol rate $f_{s}$ is $4 \mathrm{kHz}$. The DSM algorithms ((I)DSB, (I)MSDSB), discussed in [12,36], are used to solve the Green DSL problem formulations.

Impact of Channel-to-Noise Ratio A first DSL scenario is shown in Figure 5a. This is a so-called near-far ADSL downstream scenario, which is known to be challenging, where DSM can make a substantial difference. Its corresponding rate region is shown in Figure $5 \mathrm{c}$ where the blue curve is the rate region at full power and the green curve is the rate region at half power, obtained by solving DSM formulation (10) for varying weights $\mathbf{w}$ and with $\alpha$ $=1$ and $\alpha=0.5$, respectively. One can observe that saving $50 \%$ of total transmit power leads to only a small 


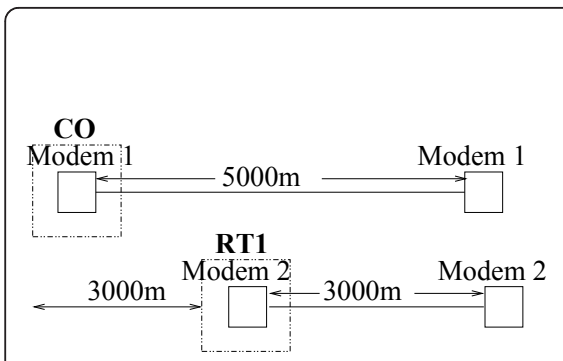

(a)

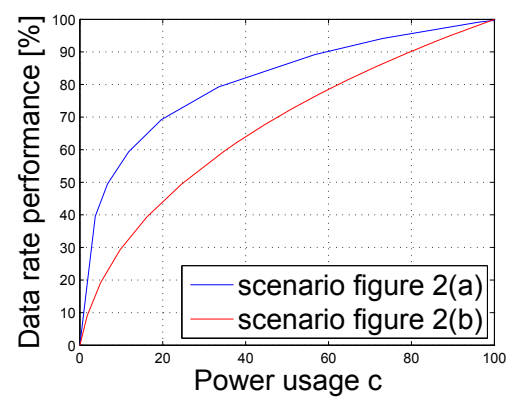

(d)

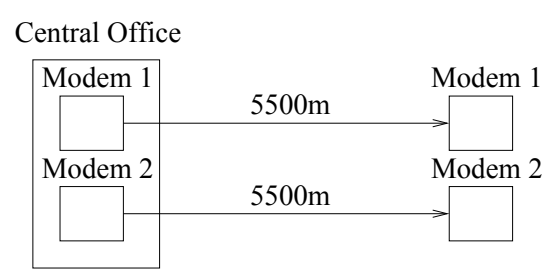

(b)

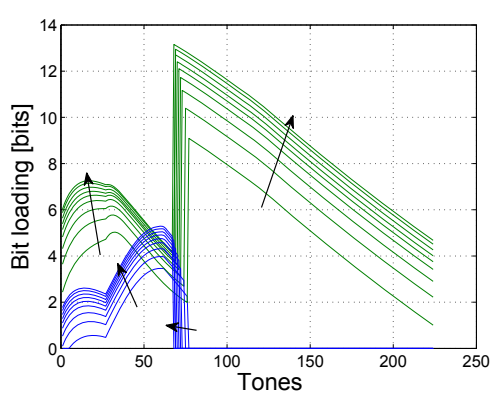

(e)

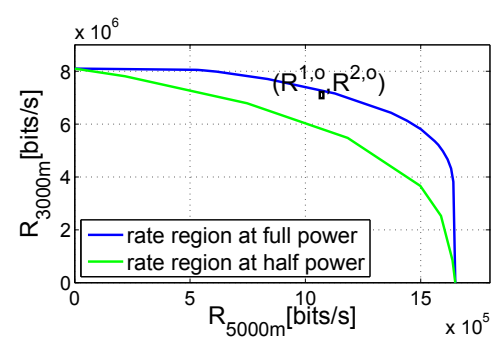

(c)

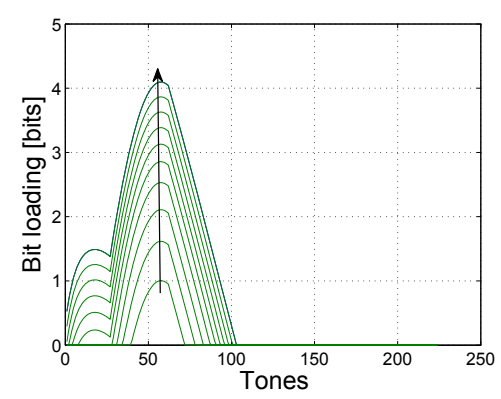

(f)

Figure $\mathbf{5}$ Simulation scenarios and results: a Near-far ADSL scenario, b Symmetric ADSL scenario, c Achievable rate region for scenario of a for different sum total powers, $\mathbf{d}$ Data rate performance in percent versus power usage for different scenarios, e Evolution of optimal bit loading for scenario of $\mathbf{a}$ for increasing powers, $\mathbf{f}$ Evolution of optimal bit loading for scenario of $\mathbf{b}$ for increasing powers.

reduction in the achievable rate region. In Figure 5d, the blue curve shows the power-rate trade-off (in percentage) for this near-far scenario. This curve is obtained by solving DSM design (8) with $t_{n}=1, n \in \mathcal{N}$, and for decreasing target rates $R^{n \text {,target }}, n \in \mathcal{N}$, i.e., $R^{n \text {,target }}=\omega \times R^{0, n}$ with $\omega=1,0.9, \ldots, 0.1,0$. One can, for instance, observe a data rate performance of $85 \%$ while saving $50 \%$ of power.

The second scenario is a symmetric downstream ADSL scenario and is depicted in Figure 5b. Its corresponding power-rate trade-off is shown as the red curve in Figure $5 \mathrm{~d}$. One can observe a data rate performance of $72 \%$ for a $50 \%$ power saving.

The evolutions of the corresponding optimal bit loadings are shown for a linearly increasing power budget ranging from 10 to $100 \%$ (i.e., full power) in steps of $10 \%$ for the two scenarios in Figure 5e, f). One can observe $a$ law of diminishing marginal returns, i.e., a linear increase in power leads to a diminishing data rate increase. Note that this effect is less obvious in Figure $5 f$, i.e., for the symmetric DSL scenario. The trade-off between data rate performance and power usage thus depends on the type of scenario.

From Theorem 4.1, we know that large CNRs result in a good power-rate trade-off, whereas small CNRs result in a linear power-rate trade-off. From Figure 5e, it can be seen that the bit loadings range from 0 to 13 bits, with an average of 6 bits. This corresponds to a rather large CNR and thus also explaining the favorable power-rate trade-off, i.e., by reducing transmit powers up to $50 \%$, only a small decrease in data rate performance of $15 \%$ is observed. In Figure $5 f$, the bit loadings, in contrary, range only between 0 to 4 bits, with an average of 2 bits. This corresponds to smaller CNRs and thus leads to a larger impact on the data rate performance, i.e., 72 for $50 \%$ power usage.

The impact on the data rate performance thus depends on the CNR, which in turn depends on the line attenuation, i.e., $\left|h_{k}^{n, n}\right|^{2}$. Note that longer lines have larger attenuations, i.e., smaller values for $\left|h_{k}^{n, n}\right|^{2}$. Thus, in scenarios with long line lengths, we will observe larger decreases in data rates for given power savings. Note, however, that the considered symmetric DSL scenario of Figure $5 \mathrm{~b}$ has a maximum line length of $5.5 \mathrm{~km}$ and is characterized by a 50-72\% power-rate trade-off. For most practical DSL scenarios, which generally have much shorter line lengths, CNRs are typically much larger, and thus, we can expect even more favorable power-rate trade-offs. This will be confirmed by further simulations in this section.

Impact of Network Size The size of the DSL network, measured by the number of interfering users, also has a significant impact on the power-rate trade-off. As explained in Section 4-B1, an increasing number of users leads to an increasing amount of crosstalk, and thus, we can expect an improvement of the power-rate trade-off. In Figure 6a, a multiuser ADSL scenario is 


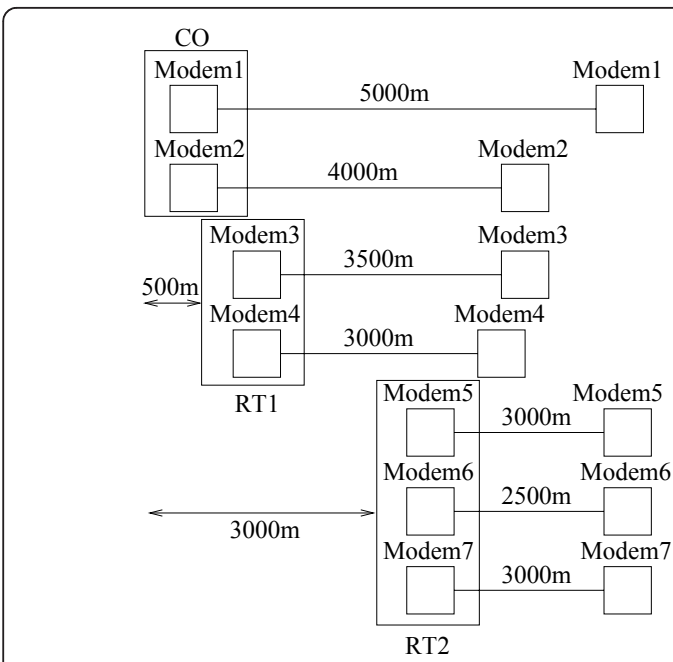

(a)

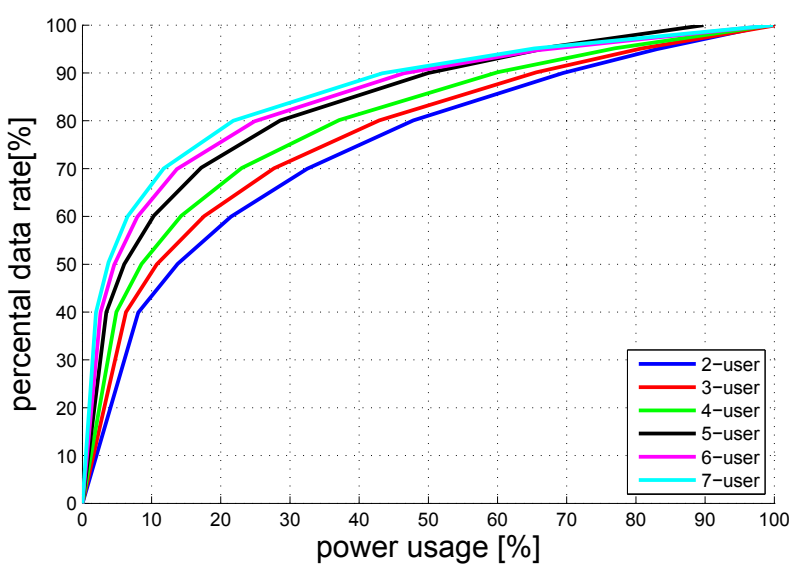

(b)

Figure 6 a N-user ADSL downstream scenario, b Trade-off data rate performance in percent versus power usage for two- up to sevenuser ADSL downstream scenario of a.

shown. For this scenario, we simulated the trade-off for the two-user case up to the seven-user case. The fouruser case, for example, consists of active modems 1, 2, 3 and 4 where modems 5,6 and 7 are inactive. The results are shown in Figure $6 \mathrm{~b}$. It can be seen that as the size of the DSL network increases, the degradation in data rate performance is smaller under given power savings. For the seven-user case, the data rate performance is $91 \%$ for $50 \%$ power usage. Note that in practice, typically 20-100 DSL lines are binded into one cable bundle, which will result in even more favorable power-rate trade-offs.

We would like to highlight here that the law of diminishing marginal returns is not just stating the concavity of the logarithmic relation between bits and powers, but there is also an additional influence by the characteristics of the crosstalk.

Impact of DSL technology Different DSL technologies $(\operatorname{ADSL}(2)(+), \operatorname{VDSL}(2))$ have different settings in terms of number of tones, band plan, power resources, line topology, average CNRs, etc. This has an influence on the studied power-rate trade-off. In Figure $7 \mathrm{a}$, a fouruser upstream VDSL scenario is shown. The corresponding trade-off is shown in Figure $7 \mathrm{~b}$. One can observe that the results are even more pronounced compared to those for the ADSL scenarios. For $50 \%$ power usage, the data rate performance is still $95 \%$ of fullpower data rate performance. Even stronger, for $20 \%$ power usage, one can still transmit at $80 \%$ of the fullpower data rate performance.

\section{CO}

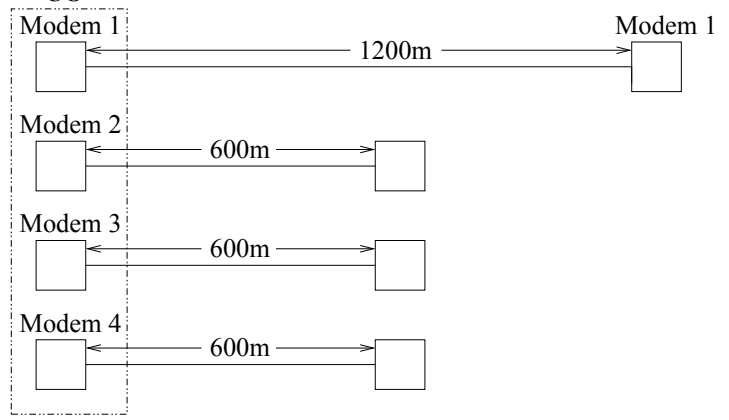

(a)

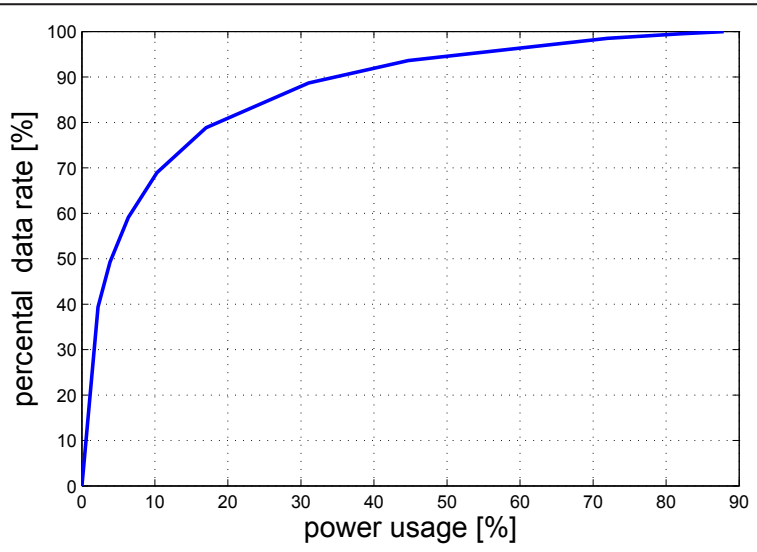

(b)

Figure 7 a VDSL upstream scenario, b Data rate performance in percent versus power usage for 4-user VDSL upstream scenario of a 
It is not so easy to make a general statement of which technology (ADSL(2) versus VDSL(2)) has a more favorable power-rate trade-off as it depends on multiple factors. Simulations, however, show that VDSL(2) systems typically have a better power-rate trade-off compared to $\operatorname{ADSL}(2)(+)$ systems. This can be explained by (i) the shorter line lengths that result in higher average CNRs and (ii) larger crosstalk levels as higher frequencies are used, which both contribute to obtain a more favorable power-rate trade-off.

Summary for price of greening dimension We can summarize that the price of greening is very small for practical DSL access networks, i.e., much better than linear. A first explanation is the typical very high CNR values of DSL copper wires. Furthermore, the presence of crosstalk in DSL systems results in an improved power-rate trade-off. In addition, newer DSL technologies such as VDSL(2) that are currently widely being deployed have better power-rate trade-offs. This means that there is a huge potential of saving a lot of transmit power in future DSL access networks by the usage of power-aware DSM.

\section{Fairness in green DSL}

It is important to define a number of greening policies, i.e., policies to green the DSL access network, which follow some fairness notion so as to prevent that some users are treated unfairly, especially for asymmetric DSL scenarios. This will be referred to as fair greening.

In this section, we propose and study different fair greening policies that have different power-rate trade-offs. These policies fit within the Green DSL framework of [26] and lead to a three-way trade-off between fast, green and fair operation, i.e., a trade-off between the price of greening, greening and the fairness of greening, respectively. This trade-off will be quantified for some concrete practical DSL scenarios.

\section{1) Fair greening policies}

There is no universally agreed notion of fairness, especially when applied in the context of an emerging topic like Green ICT. Based on a range of reasonable views on what fairness is, we develop four different greening policies for green DSL, each parametrized by a parameter $\beta$ that is used to vary the degree of greening. Throughout this subsection, for a given fair greening formulation, we denote by $\left\{\boldsymbol{R}^{o}, \boldsymbol{P}^{o}\right\}$ the rate-power operating point somewhere on the boundary of the rate region $\mathcal{R}$, i.e., the operating point before greening and that corresponds to the operating point obtained for $\beta=1$. We distinguish two strategies of integrating fairness: (1) fair greening by constraints and (2) fair greening by regularization in the objective function.

\section{Fair greening by constraints}

Here, fairness definitions are incorporated into the constraints.

\section{(1) Greening 1: Power-fair greening}

In this fair greening approach, we target "perfect power fairness" by having a weighted rate sum as the objective and proportionally reducing the available transmit power per user. This corresponds to the following parametrized optimization problem,

$$
\begin{aligned}
& \text { Greening 1: } \underset{\mathbf{s} \in \mathcal{S}}{\operatorname{maximize}} \sum_{n \in \mathcal{N}} w_{n} R^{n} \\
& \text { subject to } P^{n} \leq \beta P^{n \text {,tot }}, \quad n \in \mathcal{N},
\end{aligned}
$$

with greening parameter $\beta \in[0,1]$, where $\beta=1$ corresponds to no greening and $\beta=0$ to $100 \%$ greening. This fair greening policy can be summarized as "the degree of greening should be proportional for all users". Note that this approach does not necessarily enforce that the data rate reduction $\Delta \mathbf{R}$ in Figure 2 lies parallel with $\mathbf{R}^{o}$, i.e., not fair in the reduction in the rates.

\section{(2) Greening 2: Rate-fair greening}

In contrast to power-fair greening, this approach targets "perfect rate fairness" by having a power sum as the objective and by proportionally reducing the minimum target data rates of the users, as follows,

$$
\begin{aligned}
& \text { Greening 2: } \underset{\mathbf{s} \in \mathcal{S}}{\operatorname{maximize}}-\sum_{n \in \mathcal{N}} t_{n} P^{n} \\
& \text { subject to } P^{n} \leq P^{n, \text { tot }}, \quad n \in \mathcal{N}, \\
& R^{n} \geq \beta R^{n, o}, \quad n \in \mathcal{N},
\end{aligned}
$$

with greening parameter $\beta \in[0,1]$, where $\beta=1$ corresponds to no greening and $\beta=0$ to $100 \%$ greening. This fair greening policy can be summarized as "the price of greening should be proportional for all users". It enforces that the data rate reduction $\Delta \mathbf{R}$ in Figure 2 is parallel with $\mathbf{R}^{o}$, i.e., fair in the reduction in the rates.

\section{(3) Greening 3: Power/rate proportional greening}

In this fair greening policy, the ratios of the users' data rate decrease to their total transmit power decrease are kept the same across the users, i.e., those that transmit at higher rates take a proportionally larger share of the price of greening:

$$
\begin{aligned}
\text { Greening 3: } & \underset{\mathbf{s} \in \mathcal{S}}{\operatorname{maximize}} \sum_{n \in \mathcal{N}} w_{n} R^{n} \\
\text { subject to } P^{n} \leq P^{n, \text { tot }}, \quad n \in \mathcal{N}, & \frac{R^{n}}{R^{n, o}} / \frac{P^{n}}{P^{n, o}}=\beta, n \in \mathcal{N} .
\end{aligned}
$$

Note that this approach jointly considers fairness in data rates as well as in total transmit powers. This fair greening policy can be summarized as "the ratio of the price of greening to the degree of greening should be proportional for all users". Note that $\beta=1$ corresponds to no greening, and increasing $\beta$ corresponds to greening. One can easily extend (20) with an extra bisection search to find the value of $\beta$ that corresponds to a particular system power usage. This is because system power usage is monotonically decreasing with increasing $\beta$. 
Fair greening by regularization in objective function This type of fair greening policies implements fairness by adding a weighted fairness term to the objective.

(4) Greening 4: Weighted rate sum greening with fair regularization

Here, we start from the weighted rate sum formulation with a system total power constraint parametrized by $\beta \in$ $[0,1]$, where $\beta=1$ corresponds to no greening and $\beta=0$ to $100 \%$ greening. However, this formulation does not impose any fairness consideration and can return solutions that favor some users substantially. Therefore, the objective function is augmented with a fairness term $G^{n}$ :

$$
\text { Greening 4: } \begin{aligned}
& \sum_{n \in \mathcal{N}} w_{n} R^{n} \delta \sum_{n \in \mathcal{N}} G^{n}\left(P^{n}\right) \\
& \text { maximize } P^{n} \leq P^{n, \text { tot }}, \quad n \in \mathcal{N}, \\
& \text { subject to } \sum_{n \in \mathcal{N}} P^{n} \leq \beta \sum_{n \in \mathcal{N}} P^{n, \text { tot }},
\end{aligned}
$$

where $\delta$ is a weighting factor that can be tuned to emphasize the importance of greening fairness relative to the maximum weighted data rate performance. The function $G^{n}\left(P^{n}\right)$ may take different forms depending on the desired power fairness. One possibility is $\alpha$-fairness [31]: $G^{n}(\cdot)=(\cdot)^{1-\alpha} /(1-\alpha)$, for $\alpha>0$, and $G^{n}(\cdot)=\log (\cdot)$, for $\alpha$ $=1$, which includes max-min $(\alpha \rightarrow \infty)$ and proportional fairness $(\alpha=1)$ as special cases. Another possibility is to use a second moment as a measure of fairness: $G^{n}()=$. $(.)^{2}$. We define the following instances of this fair greening policy, which will be evaluated in Section 4-C2:

- Greening 4A: (21) with $\delta=0$;

- Greening 4B: (21) with $\delta>0$ and $G^{n}()=.-(.)^{2}$;

- Greening 4C: (21) with $\delta>0$ and $G^{n}()=.\log ($.$) .$

These fair greening policies can be summarized as "green the DSL network with or without per-user green fairness objectives installed". Note that by tuning $\delta$, we can trade-off power fairness versus weighted rate maximization. Also, note that instead of putting a fairness term in transmit powers, we can also choose a fairness term in data rates, i.e., $G^{n}\left(R^{n}\right)$ to enforce fairness over data rates if desired.

\section{2) Fair greening simulations and analysis}

The four proposed fair greening policies (Greening 1/2/ 3/4) fit within the Green DSL framework (9) and can thus be solved using the efficient algorithms as proposed in $[25,26,36]$. To demonstrate the importance of considering fairness when reducing power consumption, we have simulated the performance of the different fair greening policies for two different DSL scenarios. The first scenario is the standard near-far ADSL downstream scenario, as depicted in Figure 5a. The second scenario is an asymmetric 6-user ADSL downstream scenario, which is depicted in Figure 6a with 'modem 7' inactive.
The same realistic system parameter settings are used as in Section 4-B.

Figure 8a shows the trade-off between the normalized data rate sum performance (w.r.t. the maximum unweighted rate sum $\sum_{n} R^{n, o}$ for full power usage), and the power usage measured by the actual consumed power divided by the total maximum available power (i.e., $\sum_{n} P^{n} / \sum_{n} P^{n, \text { tot }}$ ), for the greening policies of Section 4-C1 for the 2-user DSL scenario of Figure 5a. This can be seen as the trade-off between the price of greening and the degree of greening, i.e., power-rate trade-off. One can generally observe logarithmic curves, implying that significant power savings can be achieved with only small degradations in data rate performance, as was also demonstrated in Section 4-B.

Figure $8 \mathrm{~b}$ shows the distribution of the normalized data rates (w.r.t. the full-power data rate $R^{n, o}$ for each user $n$ ) and normalized transmit powers (w.r.t. the fullpower $P^{n \text {,tot }}$ budget of each user $n$ ) across the two users for the scenario of Figure 5a for the different greening policies when $50 \%$ greening is applied, i.e., $\Sigma_{n} P^{n} / \sum_{n} P^{n,}$ ${ }^{\text {tot }}=0.5$.

It can be seen that Greening $4 \mathrm{~A}$ is the best in terms of rate sum performance, i.e., $50 \%$ of system transmit power is saved while still achieving $93 \%$ of full-power data rate performance. However, as can be seen from Figure $8 \mathrm{~b}$, Greening $4 \mathrm{~A}$ allocates the data rates and the transmit powers very unfairly over the users, i.e., user 2 dominates over user 1 in terms of transmit power as well as data rate. This unfair behavior is a result of the asymmetric interference of the considered DSL scenario and also because Greening 4A does not implement any fairness regularization, lacking any mechanism to steer toward fair transmit power and/or data rate allocations. So, in terms of Figure 2, Greening 4A has a small projected component along the price of greening dimension g, i.e., $\mathcal{P}_{\mathbf{g}}(\Delta \mathbf{R})$ is small, but a large projected component along the fairness dimension $\mathbf{f}$, i.e., $\mathcal{P}_{\mathbf{f}}(\boldsymbol{\Delta R})$ is large. This very unfair behaviour should be prevented, and it demonstrates that taking fairness into account is essential, especially for asymmetric DSL scenarios.

Greening 1 proportionally allocates the transmit powers over the users. Greening 2 equalizes the normalized data rates, but results in an uneven allocation of transmit powers. Greening $4 \mathrm{~B}$ and $4 \mathrm{C}$ succeed in obtaining relatively better fairness in terms of transmit powers as well as data rates than Greening $4 \mathrm{~A}$. This is due to the addition of the fairness term into their objective functions. Note that for Greening $4 \mathrm{~B}$ and $4 \mathrm{C}$, only one simulation point is shown rather than a parametric curve, due to the dependence on the chosen weight $\delta$ in (21). Different values for this weight lead to different trade-offs between data rate performance and fairness in transmit powers, where we have tuned the weight to 


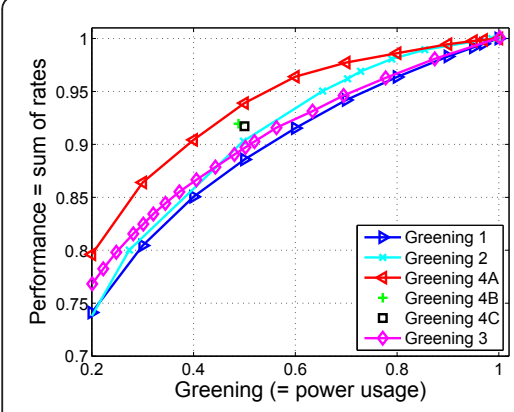

(a)
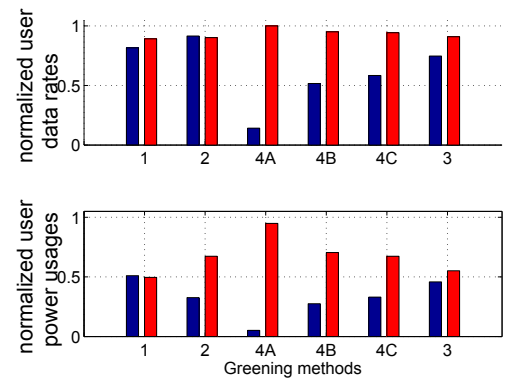

(b)

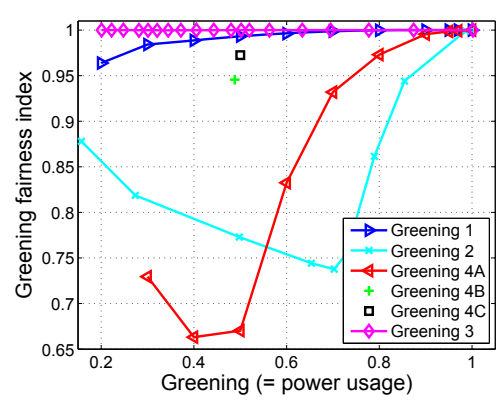

(c)

Figure 8 Fair greening simulation results for two-user DSL scenario of Fig. 5a: a Normalized data rate sum performance versus greening for different greening policies, b Normalized data rates (top) and normalized transmit powers (bottom) for two modems (blue: modem 1, red: modem 2) for different greening policies when $50 \%$ greening is applied, c Greening fairness index $\mathcal{F}$ (22) versus greening for different greening policies. For Greening $4 \mathrm{~B}$ and $4 \mathrm{C}$, the values $\delta=410^{5}$ and $\delta=90$ were used, respectively. Weights $w_{n}, n \in \mathcal{N}$, are fixed at 1.

obtain a good trade-off between both objectives. Finally, Greening 3 results in a ratio of the normalized data rates to the normalized transmit powers that is proportional for all users.

The inclusion of fairness thus results in a fairer allocation of data rates and/or transmit powers, but the price of adding fairness is a reduced data rate sum performance compared to Greening 4A, as can be seen in Figure 8a.

Fairness metric To further quantitatively evaluate the fairness of the greening policies, a fairness measure is needed. Different scalar-valued measures of fairness can be chosen, which focus on data rates or powers. A good candidate is a measure that jointly considers power and data rate fairness in its definition and is designed for the considered specific case of greening in interference-limited networks. To this end, we propose the following definition of the greening fairness index $\mathcal{F}$ :

$$
\mathcal{F}=\frac{1}{N-1}\left(\frac{\left(\sum_{n \in \mathcal{N}} x_{n}\right)^{2}}{\sum_{n \in \mathcal{N}} x_{n}^{2}}-1\right),
$$

with $x_{n}=\left(R^{n} / R^{n, o}\right) /\left(P^{n} / P^{n, o}\right)$ and $\left\{\mathbf{R}^{o}, \mathbf{P}^{o}\right\}$ denotes the point on the boundary of the rate region without greening. $\mathcal{F}=1$ when all users have the same ratio between data rate decrease and power usage decrease and approaches zero as these ratios start to deviate from each other. Figure $8 \mathrm{c}$ shows the trade-off between the greening fairness index $\mathcal{F}$ and the power usage for the proposed fair greening policies. The key messages are as follows:

- Power/Rate Proportional Greening 3 is 100\% fair w.r.t. $\mathcal{F}$, since it was constructed by considering both power usage and data rate in the first place.

- Power-fair Greening 1 is also quite fair, whereas rate-fair Greening 2 is unfair.
- Weighted rate sum Greening 4A is very unfair. However, by adding the fairness terms to Greening $4 \mathrm{~A}$, i.e., Greening $4 \mathrm{~B}$ and $4 \mathrm{C}$, the fairness behavior, w.r.t. $\mathcal{F}$, becomes much better, i.e., from $67 \%$ to more than $94 \%$.

The same graphs are also simulated for the 6-user ADSL scenario of Figure 6a and are depicted in Figure $9 \mathrm{a}, \mathrm{b}, \mathrm{c}$. Note that for these simulations we have used the following weights $\mathbf{w}=[0.2,0.2,0.2,0.2,0.1,0.1]^{\mathrm{T}}$ so as to obtain a working point for which the data rates of the short lines, i.e., line 5 and 6 , are within the data rate range of the longer lines, i.e., line 1, 2, 3 and 4. It can be observed that Greening 4A results in the best powerrate trade-off. However, it also corresponds to very unfair transmit power distributions where users 5 and 6 reduce their data rates and especially their total transmit powers much more than the other users. Greening 4B and $4 \mathrm{C}$ improve this unfair behavior from 43 to 70 and $80 \%$, respectively. Greening 2 is $100 \%$ rate fair but has an overall unfair behavior of $54 \%$. This is caused by its very unfair transmit power distribution. Both Greening 1 and 3 have a very good fairness performance, but this comes with a price of greening, i.e., a decrease in normalized weighted data rate sum performance of 4 and $4.5 \%$, respectively.

The key messages can thus be summarized as follows:

- Fairness should be considered carefully so as to prevent that some users are treated unfairly or even put out-of-service.

- Greening 1, i.e., proportionally decreasing all users' transmit powers, generally leads to a good fairness behavior, which is much better than Greening 2, i.e., proportionally decreasing the data rates for all modems. 
- Greening 3 results in a behavior that is jointly fair in data rate and transmit power allocations.

- The inclusion of fairness comes with a price in data rate performance.

Trade-off Fast versus Fair versus Green To quantify the trade-off among fast, fair and green operation, we introduce the concept of the fast-fair-green (FFG) triangle. The FFG triangle is depicted in Figure 10a, b. It consists of three axes that indicate the level of fast, fair or green operation, ranging from 0 to $100 \%$. The particular definitions for each of the axes can vary depending on the desired three-way trade-off. For instance, $100 \%$ fast operation can correspond to a working point on the boundary of the rate region, whereas $0 \%$ fast operation can correspond to a zero data rate working point. $0 \%$ green operation can correspond to the case where all users consume full power, i.e., $P^{n}=P^{n \text {, tot }}, n \in \mathcal{N}$, whereas $100 \%$ green corresponds to no power usage. For the fairness axis one may use some notion of fairness.

We have used the following definitions to quantify the three-way trade-off for the proposed fair greening policies:

- FAST: $\frac{\sum_{n \in \mathcal{N}} w_{n} R^{n}}{\sum_{n \in \mathcal{N}} w_{n} R^{n, o}}$

- FAIR: $\mathcal{F}$ (= fair greening index (22))

- GREEN: $1-\frac{\sum_{n \in \mathcal{N}} P^{n}}{\sum_{n \in \mathcal{N}} P^{n, o}}$

Note that, with these definitions, it is impossible to achieve a triangle that is both $100 \%$ fast and $100 \%$ green. In Figure 10a, b, we have plotted the FFG triangles for 'No greening', Greening 4A and Greening 3 for 50\% greening for the 2-user DSL scenario of Figure $5 \mathrm{a}$ and the 6-user DSL scenario of Figure 6a, respectively. 'No greening' corresponds to the working point on the boundary of the rate region. It can be seen that Greening 3 achieves a considerable better three-way trade-off w.r.t Greening 4A and 'No greening'.

We can now define a joint FFG performance measure to quantify the FFG trade-off performance for the different greening policies. One possibility would be to take the average of the performances along the three axes. This linear combination however, does not distinguish between symmetric and asymmetric FFG performances such as $F / F / G=0.8 / 0.8 / 0.8$ and $F / F / G=1.0 / 1.0 / 0.4$, respectively. We want the joint performance measure to reflect that solutions with small performances along either of the axes are less desired. Therefore, a better option would be to use a product joint performance measure, i.e., $F \times F \times G$. The 'No greening' solution would then, however, correspond to a zero performance value because $G=0$. Therefore, we choose to define the joint performance measure as the ratio of the area of the FFG triangle to the area of the full triangle obtained by the points (100\% fast, $100 \%$ green and $100 \%$ fair). This joint performance measure favors symmetric FFG trade-off performances more than asymmetric ones and furthermore does not necessarily result in a zero-value if the performance along one of the axes is zero. The results for this joint performance measure are shown in Table 1 for the 2-user case and the 6-user case, respectively.

One can see that Greening 3 results in a very good FFG trade-off performance. For the 2-user case, it performs $84.13 \%$ better than 'No greening' and $28.39 \%$ better than Greening 4A. For the 6-user case, Greening 3 performs $80.53 \%$ better than 'No greening' and $66.77 \%$ better than Greening 4A. One can notice that Greening 1 also results in a very good FFG trade-off performance. This means that the proportional reduction in the available transmit powers results in a quite reasonable proportional data rate reduction, and so Greening 1 can be seen as a good fair greening policy. In contrast, Greening 2 results in a

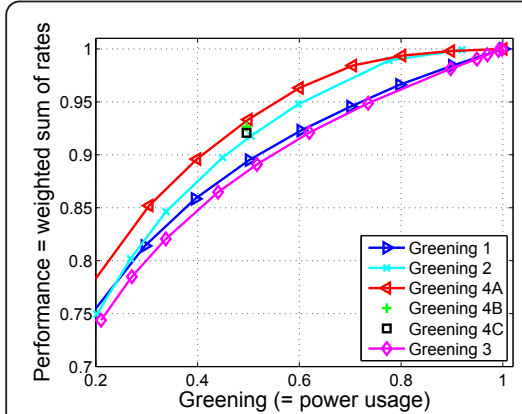

(a)
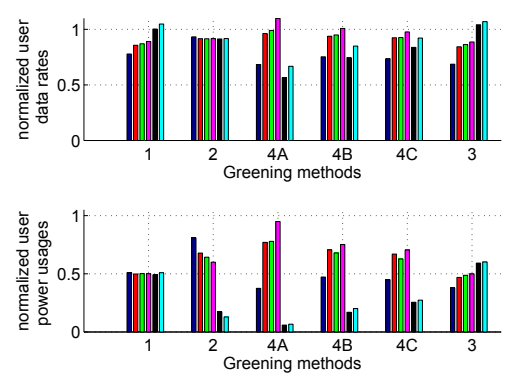

(b)

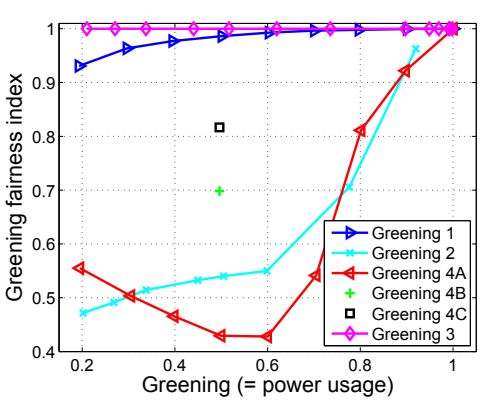

(c)

Figure 9 Fair greening simulation results for six-user DSL scenario of Fig. 6a: a Normalized sum data rate performance versus greening for different greening policies, $\mathbf{b}$ Normalized data rates (top) and normalized transmit powers (bottom) for six modems (different colors with user 1-6 from left to right) for different greening policies when $50 \%$ greening is applied, c Greening fairness index $\mathcal{F}$ (22) versus greening for different greening policies. For Greening $4 \mathrm{~B}$ and $4 \mathrm{C}$, the values $\delta=410^{5}$ and $\delta=90$ were used, respectively. 


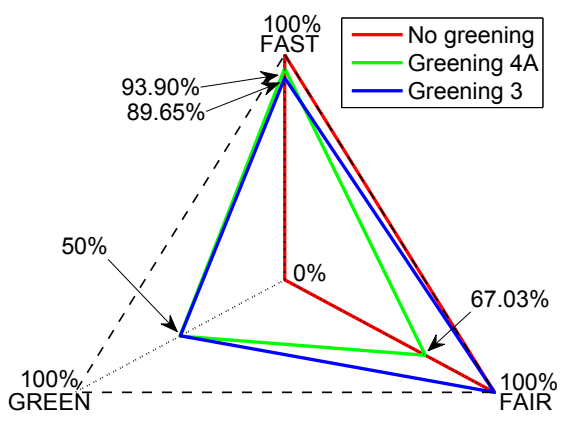

(a) 2-user ADSL

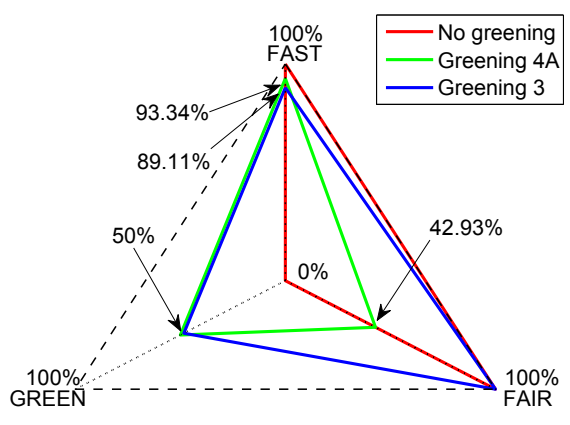

(b) 6-user ADSL

Figure 10 Fast-fair-green triangles for different fair greening policies and for different DSL scenarios

much less favorable FFG trade-off. Greening 4B and 4C have a better FFG trade-off performance with respect to Greening 4A, which is a result of the inclusion of the per-user fairness objectives $G^{n}($.).

\section{Conclusion}

Reducing the power consumption of broadband access networks has gained quite some momentum over the last few years. Dynamic spectrum management (DSM) is a very promising technique to significantly improve the power efficiency of DSL broadband access networks by optimizing the transmit powers. In this paper, we have

Table 1 FFG trade-off performances for different fair greening policies for $\mathbf{5 0 \%}$ greening.

\begin{tabular}{lcc}
\hline & 2-user ADSL (Fig. 5a) (\%) & 6-user ADSL (Fig. 6a) (\%) \\
\hline No greening & 33.33 & 33.33 \\
Greening 1 & 60.65 & 60.69 \\
Greening 2 & 51.30 & 40.57 \\
Greening 4A & 47.80 & 36.08 \\
Greening 4B & 60.80 & 48.85 \\
Greening 4C & 61.23 & 54.24 \\
Greening 3 & 61.37 & 60.17 \\
\hline
\end{tabular}

first proposed some novel power- and energy-aware DSM problem formulations that fit within our recently proposed 'Green DSL' framework [25,26] and that improve the power efficiency of DSL transmission. However, the formulation of these power-aware DSM formulations requires a proper understanding of greening in interference-limited networks. We have, therefore, investigated the concept of greening and have identified that greening can be decomposed into two essential greening dimensions: (1) the price of greening, i.e., the powerrate trade-off, and (2) the fairness of greening. Only by addressing both dimensions, one can obtain proper greening strategies. Along both dimensions, we have conducted a thorough analysis consisting of theoretical results and also simulation-based results so as to identify the typical trends that can be expected for practical DSL scenarios under different realistic settings. We have shown that the power-rate trade-off depends on the CNRs, the available power budgets, the DSL technology, as well as the interference characteristics. Furthermore, we have shown that very large power savings can be obtained with only a minor impact on the data rate performances. For instance, we have demonstrated that for some VDSL scenarios transmit power savings of up to $50 \%$ can be achieved while preserving $95 \%$ of full-power data rate performance. Finally, we have identified that fairness is a very important dimension that has to be taken into account to prevent unfair allocation when greening, especially in asymmetric DSL scenarios. Therefore, we have proposed four different fair greening policies to incorporate fairness when greening, of which Greening 3 achieves a good trade-off between fast, fair and green operation.

\section{Appendix}

\section{A. Proof of (13) being a special case of (8)}

Proof: As $E_{n}=P^{n} T^{n}$ and $T^{n}=J^{n} / R^{n}$, (13) can be reformulated as follows

$$
\begin{aligned}
\underset{s \in \mathcal{S}}{\operatorname{minimize}} & \sum_{n \in \mathcal{N}} J^{n} P^{n} / R^{n} \\
\text { subject to } & R^{n} \geq J^{n} / T^{n, \text { target }}, \quad n \in \mathcal{N}, \\
& P^{n} \leq P^{n, \text { tot }, \quad n \in \mathcal{N} .}
\end{aligned}
$$

As $R^{n}\left(P^{n}\right)$ is concave in $P^{n}$ for the multi-user case (see Section 4-B1), $R^{n}\left(P^{n}\right) / P^{n}$ is maximized when $P^{n} \rightarrow$ 0 or equivalently $R^{n}\left(P^{n}\right) \rightarrow 0$. Reducing $P^{n}$, and the corresponding $R^{n}\left(P^{n}\right)$, results in a decrease of $\sum_{m \neq n} P^{m}$ $J^{m} / R^{m}$ as the data rates of the other users are increased because less crosstalk is radiated to them. The optimal solution thus corresponds to minimizing powers and rates for all users, or equivalently maximizing the times to finish the jobs, which results in the equality $T^{n}=T^{n \text {, }}$ target. This proofs that (13) is special case of (8) with $t_{n}=T^{n, \text { target }}$ and $R^{n, \text { target }}=J^{n} / T^{n, \text { target }}$. 


\section{B. Proof of Lemma 4.1}

Proof: For a single-user case, it is known that the optimal transmit powers satisfy the following water-filling Karush-Kuhn-Tucker (KKT) conditions [4]

$$
\begin{aligned}
& s_{k}^{n, *}=\frac{w_{n}}{\lambda_{n}^{*} \log (2)}-\frac{\Gamma \sigma_{k}^{n}}{\left|h_{k}^{n, k}\right|^{2}}, \quad k \in \mathcal{K}, \\
& \sum_{k \in \mathcal{K}} s_{k}^{n, *}=P^{n, \text { tot }}, \quad s_{k}^{n, *} \geq 0, \quad k \in \mathcal{K},
\end{aligned}
$$

with $s_{k}^{n, *}$ and $\lambda_{n}^{*}$ being the optimal transmit powers and Lagrange multiplier, respectively, for a total transmit power consumption $P^{n \text {, tot }}$. Similarly, we can define the optimal transmit powers for the reduced total power $c$. $P^{n, \text { tot }}$ as follows

$$
\begin{aligned}
& s_{k}^{n, *, c}=\frac{w_{n}}{\lambda_{n}^{c, *} \log (2)}-\frac{\Gamma \sigma_{k}^{n}}{\left|h_{k}^{n, n}\right|^{2}}, \quad k \in \mathcal{K}, \\
& \sum_{k \in \mathcal{K}} s_{k}^{n, *, c}=c \cdot P^{n, \text { tot }}, \quad s_{k}^{n, *, c} \geq 0, \quad k \in \mathcal{K},
\end{aligned}
$$

with $s_{k}^{n, *, c}$ and $\lambda_{n}^{*, c}$ the optimal transmit powers and Lagrange multiplier, respectively, for a total transmit power consumption $c \cdot P^{n \text {,tot }}$. From (24) and (25), we can obtain the following equation

$$
\frac{w_{n}}{\lambda_{n}^{*} \log (2)}=\left(P^{n, \text { tot }}+\sum_{k \in \mathcal{K}} \frac{\Gamma \sigma_{k}^{n}}{\left|h_{k}^{n, n}\right|^{2}}\right) \frac{1}{K} .
$$

Similarly, we can obtain from (26) and (27) the following equation

$$
\frac{w_{n}}{\lambda_{n}^{*, c} \log (2)}=\left(c \cdot P^{n, \text { tot }}+\sum_{k \in \mathcal{K}} \frac{\Gamma \sigma_{k}^{n}}{\left|h_{k}^{n, n}\right|^{2}}\right) \frac{1}{K} .
$$

The relative data rate reduction for a total transmit power of $c \cdot P^{n \text {, tot }}$ can be expressed as follows

$$
f(c)=\frac{R^{n}\left(c \cdot P^{n, \text { tot }}\right)}{R^{n}\left(P^{n, \text { tot }}\right)}=\frac{\sum_{k \in \mathcal{K}} \log _{2}\left(1+s_{k}^{n, c, *} \frac{\left|h_{k}^{n, n}\right|^{2}}{\Gamma \sigma_{k}^{n}}\right)}{\sum_{k \in \mathcal{K}} \log _{2}\left(1+s_{k}^{n, *} \frac{\left|h_{k}^{n, n}\right|^{2}}{\Gamma \sigma_{k}^{n}}\right)}
$$

From (24), (26), (28) and (29), this can be rewritten as follows

$$
f(c)=\frac{\sum_{k \in \mathcal{K}} \log _{2}\left(\frac{c}{K} P^{n, \text { tot }} \cdot \mathrm{CNR}_{k}^{n}+\frac{1}{K} \sum_{q \in \mathcal{K}} \frac{\mathrm{CNR}_{k}^{n}}{\mathrm{CNR}_{q}^{n}}\right)}{\sum_{k \in \mathcal{K}} \log _{2}\left(\frac{1}{K} P^{n, \text { tot }} \cdot \mathrm{CNR}_{k}^{n}+\frac{1}{K} \sum_{q \in \mathcal{K}} \frac{\mathrm{CNR}_{k}^{n}}{\mathrm{CNR}_{q}^{n}}\right)}
$$

\section{Acknowledgements}

Paschalis Tsiaflakis is a postdoctoral fellow funded by the Fonds Wetenschappelijk Onderzoek (FWO) - Vlaanderen. This research work was carried out at the ESAT Laboratory of Katholieke Universiteit Leuven, in the frame of K.U. Leuven Research Council CoE EF/05/006 Optimization in Engineering (OPTEC), Concerted Research Action GOA-MaNet, the Belgian Programme on Interuniversity Attraction Poles initiated by the Belgian Federal Science Policy Office IUAP P6/04 (DYSCO, Dynamical systems, control and optimization, 2007-2011), Research Project FWO nr.G.0235.07 (Design and evaluation of DSL systems with common mode signal exploitation), and is supported in part by Princeton Grand Challenge Grant, NSF NetSE grant on Socio-Technical Networking and a Google grant, and also by Basic Science Research Program through the National Research Foundation of Korea (NRF) funded by the Ministry of Education, Science and Technology (2011-0015042). A part of this paper has appeared in the Proceedings of the IEEE International Conference on Communications (ICC), Dresden, Germany, June 2009 [25] and in the Proceedings of the ACM GreenMetrics Workshop, Seattle, WA, June 2009 [26].

\section{Author details}

'Department of Electrical Engineering, ESAT/SCD, Katholieke Universiteit Leuven, Kasteelpark Arenberg 10, 3001 Leuven-Heverlee, Belgium ${ }^{2}$ Department of Electrical Engineering, KAIST, Building E3, 6-3205, Republic of Korea ${ }^{3}$ Department of Electrical Engineering, Princeton University, B328, Engineering Quad, Olden Street, Princeton, NJ 08544, USA

\section{Competing interests}

The authors declare that they have no competing interests.

Received: 3 February 2011 Accepted: 26 October 2011

Published: 26 October 2011

\section{References}

1. F Vanier, World broadband statistics: Q1 2009, Technical report, Point Topic Ltd, (2009) http://www.point-topic.com

2. KB Song, ST Chung, G Ginis, JM Cioffi, Dynamic spectrum management for next-generation DSL systems. IEEE Commun Mag. 40(10), 101-109 (2002). doi:10.1109/MCOM.2002.1039864

3. JM Cioffi., et al, Vectored DSLs with DSM: the road to ubiquitous gigabit DSLs. Proceedings of the World Telecommunications Congress Budapest 1-11 (2006)

4. W Yu, G Ginis, JM Cioffi, Distributed multiuser power control for digital subscriber lines. IEEE J Sel Areas Commun. 20(5), 1105-1115 (2002). doi:10.1109/JSAC.2002.1007390

5. R Cendrillon, W Yu, M Moonen, J Verlinden, T Bostoen, Optimal multiuser spectrum balancing for digital subscriber lines. IEEE Trans Commun. 54(5), 922-933 (2006)

6. Y Xu, T Le-Ngoc, S Panigrahi, Global concave minimization for optimal spectrum balancing in multi-user DSL networks. IEEE Trans Signal Process. 56(7), 2875-2885 (2008)

7. P Tsiaflakis, J Vangorp, M Moonen, J Verlinden, A low complexity optimal spectrum balancing algorithm for digital subscriber lines. Signal Process. 87(7), 1735-1753 (2007). doi:10.1016/j.sigpro.2007.01.020

8. R Cendrillon, J Huang, M Chiang, M Moonen, Autonomous spectrum balancing for digital subscriber lines. IEEE Trans Signal Process. 55(8), 4241-4257 (2007)

9. W Yu, Multiuser water-filling in the presence of crosstalk. Proceedings of the Information Theory and Application (2007)

10. RB Moraes, B Dortschy, A Klautau, JR i Riu, Semiblind spectrum balancing for DSL. IEEE Trans Signal Process. 58(7), 3717-3727 (2010)

11. S Huberman, C Leung, T Le-Ngoc, Dynamic spectrum management (DSM) algorithms for multi-user XDSL. submitted to IEEE Commun Surv Talks (2009)

12. P Tsiaflakis, M Diehl, M Moonen, Distributed spectrum management algorithms for multiuser DSL networks. IEEE Trans Signal Process. 56(10), 4825-4843 (2008)

13. $\mathrm{W} Y \mathrm{Yu}, \mathrm{JM}$ Cioffi, Multiuser detection in vector multiple access channels using generalized decision feedback equalization, in Proceedings of the 5 th International Conference on Signal Processing, World Computer Congress. 20, 1771-1777 (2000)

14. G Ginis, JM Cioffi, Vectored transmission for digital subscriber line systems. IEEE J Sel Areas Commun. 20(5), 1085-1104 (2002). doi:10.1109/ JSAC.2002.1007389 
15. R Cendrillon, G Ginis, E Van Den Bogaert, M Moonen, A near-optimal linear crosstalk canceler for upstream VDSL. IEEE Trans Signal Process. 54(8), 3136-3146 (2006)

16. R Cendrillon, G Ginis, E Van Den Bogaert, M Moonen, A near-optimal linear crosstalk precoder for downstream VDSL. IEEE Trans Commun. 55(5), 860-863 (2007)

17. ITU-T Study Group 15, 2008 ICTs and Climate Change: ITU background report http://www.itu.int/dms_pub/itu-t/oth/09/05/T09050000010001PDFE. pdf (2008)

18. European Commission DG JRC Paolo Bertoldi, 2008 European Code of Conduct for Broadband Equipment http://www.itu.int/dms_pub/itu-t/oth/ 09/05/T09050000010004PDFE.pdf (2008)

19. Les Humphrey BT, UK, Opportunities and techniques for power saving in DSL http://www.itu.int/dms_pub/itu-t/oth/09/05/T09050000010003PDFE.pdf (2008)

20. M Wolkerstorfer, D Statovci, T Nordström, Dynamic spectrum management for energy-efficient transmission in DSL, in Proceedings of the 11th IEEE International Conference on Communication System, 1015-1020 (2008)

21. T Nordström, M Wolkerstorfer, D Statovci, Energy efficient power back-off management for VDSL2 transmission, in Proceedings of the European Signal Processing Conference (EUSIPCO) Glasgow, 2097-2101 (2009)

22. M Monteiro, N Lindqvist, A Klautau, Spectrum balancing algorithms for power minimization in DSL networks, in Proceedings of the IEEE International Conference on Communication, 1-5 (2009)

23. M Guenach, C Nuzman, J Maes, M Peeters, On power optimization in DSL systems, in Proceedings of the IEEE International Conference on Communication (Dresden, 2009), pp. 1-5

24. R Mohammadian, M Biguesh, S Gazor, A power allocation method for DMTbased DSL systems using geometric programming. IEEE Signal Process Lett. 17(1), 16-19 (2010)

25. P Tsiaflakis, Y Yi, M Chiang, M Moonen, Green DSL, Green DSL: Energyefficient DSM, in Proceedings of the IEEE Intetnational Conference on Communications, 1-5 (2009)

26. P Tsiaflakis, Y Yi, M Chiang, M Moonen, Fair greening for DSL broadband access, in Proceedings of the Green Metrics Workshop ACM Sigmetrics/ Performance (Seattle, Washington, 2009), pp. 1-5

27. JM Cioffi, S Jagannathan, W Lee, H Zou, A Chowdhery, W Rhee, G Ginis, P Silverman, Greener copper with dynamic spectrum management, in Proceedings of the AccessNets (Las Vegas) (2008)

28. W Yu, T Lan, Transmitter optimization for the multi-antenna downlink with per-antenna power constraints. IEEE Trans on Signal Process. 55(6), 2646-2660 (2007)

29. M Mohseni, R Zhang, JM Cioffi, Optimized transmission for fading multipleaccess and broadcast channels with multiple antennas. IEEE J Sel Areas Commun. 24(8), 1627-1639 (2006)

30. T Lan, D Kao, M Chiang, A Sabharwal, An axiomatic theory of fairness for resource allocation. in Proceedings of the IEEE INFOCOM (San Diego) (2010)

31. J Mo, J Walrand, Fair end-to-end window-based congestion control. IEEE/ ACM Trans Netw. 8(5), 556-567 (2000). doi:10.1109/90.879343

32. T Starr, JM Cioffi, PJ Silverman, Understanding Digital Subscriber Lines, (Prentice Hall, 1999)

33. W Yu, R Lui, Dual methods for nonconvex spectrum optimization of multicarrier systems. IEEE Trans Commun. 54(7), 1310-1322 (2006)

34. X Lin, NB Shroff, R Srikant, A tutorial on cross-layer optimization in wireless networks. IEEE J Sel Areas Commun. 24(8), 1452-1463 (2006)

35. L Georgiadis, MJ Neely, L Tassiulas, Resource Allocation and Crosslayer Control in Wireless Networks, vol. 1. (Foundations and Trends in Networking, 2006)

36. P Tsiaflakis, I Necoara, JAK Suykens, M Moonen, Improved dual decomposition based optimization for DSL dynamic spectrum management. IEEE Trans Signal Process. 58(4), 2230-2245 (2010)

doi:10.1186/1687-1499-2011-140

Cite this article as: Tsiaflakis et al:. Fair greening of broadband access: spectrum management for energy-efficient DSL networks. EURASIP Journal on Wireless Communications and Networking 2011 2011:140.

\section{Submit your manuscript to a SpringerOpen ${ }^{\circ}$ journal and benefit from:}

- Convenient online submission

- Rigorous peer review

- Immediate publication on acceptance

- Open access: articles freely available online

- High visibility within the field

- Retaining the copyright to your article

Submit your next manuscript at $\gg$ springeropen.com 